\title{
Quantum Group Symmetries and Non-Local Currents in 2D QFT
}

\author{
Denis Bernard ${ }^{1}$ and André LeClair ${ }^{2}$ \\ ${ }^{1}$ Service de Physique Théorique de Saclay, F-91191 Gif-sur-Yvette, France \\ 2 Newman Laboratory of Nuclear Studies, Cornell University, Ithaca, NY 14853, USA
}

Received November 13, 1990

\begin{abstract}
We construct and study the implications of some new non-local conserved currents that exist is a wide variety of massive integrable quantum field theories in 2 dimensions, including the sine-Gordon theory and its generalization to affine Toda theory. These non-local currents provide a non-perturbative formulation of the theories. The symmetry algebras correspond to the quantum affine Kac-Moody algebras. The $S$-matrices are completely characterized by these symmetries. Formal $S$-matrices for the imaginary-coupling affine Toda theories are thereby derived. The application of these $S$-matrices to perturbed coset conformal field theory is studied. Non-local charges generating the finite dimensional Quantum Group in the Liouville theory are briefly presented. The formalism based on non-local charges we describe provides an algernative to the quantum inverse scattering method for solving integrable quantum field theories in $2 \mathrm{~d}$.
\end{abstract}

\section{Table of Contents}

1. Introduction . . . . . . . . . . . . . . . . . . . 100

2. Non-Local Charges in $2 \mathrm{D}$ QFT . . . . . . . . . . . . . 102

2a. General Theory . . . . . . . . . . . . . . . . . 102

2b. The Algebra of Charges in Perturbed CFT . . . . . . . . . . 105

3. Non-Local Charges in the Sine-Gordon Theory . . . . . . . . . . . 106

3a. A Review of the Sine-Gordon Theory . . . . . . . . . . . . . . . 107

3b. The Non-Local Conserved Charges . . . . . . . . . . . . . . . 108

3c. The Algebra of the Non-Local Charges . . . . . . . . . . . . . 110

3d. The Fundamental Soliton Fields . . . . . . . . . . . . . . 111

3e. The Representation of the Non-Local Charges on Asymptotic Multi-Soliton States . . . . . . . . . . . . . . . 113

3f. The $S$-Matrix from the Non-Local Charges . . . . . . . . 115 
3g. Alternative Descriptions: The Deformed Gross-Neveu Models . . . 116

3h. The Restricted Sine-Gordon Theory Revisited . . . . . . . . . 118

3i. Additional Remarks . . . . . . . . . . . . . . . . . . . . . 120

4. Introducing the Center: The Central Sine-Gordon Models . . . . . . . 121

5. Generalization to Other Groups . . . . . . . . . . . . . . 122

5a. Non-Local Charges for the Affine Toda Theories . . . . . . . . 122

5b. The Soliton Fields and the S-Matrices _ . . . . . . . . . . 124

5c. The Gross-Neveu Limit . . . . . . . . . . . . . . . . . . . . 127

5d. Application to Perturbed Coset Conformal Field Theories . . . . 129

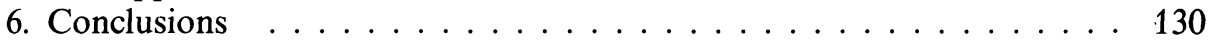

Appendix A: Quantum Affine Algebras . . . . . . . . . . . . . 131

Appendix B: Details on the Sine-Gordon S-Matrix . . . . . . . . . . 132

Appendix C: The Yangian Limit . . . . . . . . . . . . . . 133

Appendix D: Generalized Parafermions and Their Braidings . . . . . 136

\section{Introduction}

Symmetry in quantum field theory is widely recognized as being of fundamental importance. In $3+1$ spacetime dimensions, the likely symmetries of the $S$-matrix are subject to the severe limitations of the Coleman-Mandula theorem [1]. The theorem states that any symmetry group is necessarily locally isomorphic to the direct product of an internal symmetry group and the Poincaré group. These possible symmetries are normally not restrictive enough to allow a non-perturbative solution of the theory.

In lower-dimensional quantum field theory, some of the postulates of the Coleman-Mandula theorem may be relaxed in a non-trivial way. Apart from the structure of the Poincaré group itself, one of the main assumptions of the theorem is that the symmetry group acts on multi-particle states as if they were tensor products of one-particle states. More specifically, let $Q$ be a generator of the symmetry group and $V$ denote the Hilbert space of one-particle states. The multi-particle states are spanned by $V \otimes \cdots \otimes V$. The action of the generator on a multi-particle state is an operator from $V \otimes \cdots \otimes V$ into itself, and will be referred to as the comultiplication $\Delta(Q)$. The proof of the Coleman-Mandula theorem assumed the trivial comultiplication:

$$
\Delta(Q)=Q \otimes 1 \otimes \cdots \otimes 1+1 \otimes Q \otimes 1 \otimes \cdots \otimes 1+\cdots+1 \otimes \cdots \otimes Q .
$$

Supersymmetry in any number of dimensions is of course a well known example of how the hypothesis (1.1) can be circumvented.

This paper is primarily devoted to the construction of some new quantum symmetries that exist is a wide variety of integrable quantum field theories in $1+1$ dimensions. The conserved currents that generate the symmetries are non-local and further characterized by non-trivial equal-time commutation, or braiding, relations. These exceptional properties of the currents are responsible for the non-trivial comultiplication of the charges when acting on multi-particle states. That is, the assumption (1.1) of the Coleman-Mandula theorem is violated.

The existence of a non-trivial comultiplication for the non-local conserved charges implies that they belong to the algebraic framework of Hopf algebras, 
notably the Quantum Groups and the Yangian symmetries [2-4]. Our work thus provides explicit currents that generate the Quantum Group Symmetries. The non-local charges for the Yangian symmetry were studied in [5-7]. For the case of the sine-Gordon (SG) theory, the symmetry algebra is the quantum sl(2) loop algebra, which is an infinite dimensional symmetry. This algebra can be thought of as a generalization of $N=2$ supersymmetry, and is in fact equivalent to it for a special value of the $\mathrm{SG}$ coupling constant, as we will see.

The sine-Gordon theory can be completely reformulated using the non-local conserved charges. This leads to a new, remarkably simple derivation of the soliton $S$-matrix. Though the final result is known [8], the steps leading to it provide interesting non-perturbative information. Specifically, the derivation of the $S$-matrix allows us to determine which fields create the SG solitons within our framework. Our conclusions are somewhat unexpected in that the fundamental soliton fields can only be identified with the Thirring fermion fields at the point where the Thirring model is free (see $[9,10])$.

The framework we develop for the sine-Gordon theory is readily generalized to incorporate the affine Toda theories over a simply-laced group $G$. Though the action of the affine Toda theories is ill-defined, the non-local charges provide a formal non-perturbative definition of the models. In particular we are able to derive the formal $S$-matrices for these theories at imaginary coupling constant. This result can then be applied to massive perturbations of the coset conformal field theories, with the aim of establishing the conjectured form $[11,12]$ of the $S$-matrices.

The subject of integrable quantum field theory has a long history, with many impressive results. The Leningrad school developed the quantum inverse scattering method (QISM) to quantize integrable systems, including the SG model $[13,14]$. It was this investigation that led to the theory of the Quantum Group. In quantum field theory the QISM was developed as a way to quantize the theory in a manner that preserves the infinite number of commuting integrals of motion which exist in the classical theory. It is of interest to compare the QISM to the framework advocated in this paper. Contrary to the QISM, the non-local charge framework deals with conserved charges that are not in involution. The strength of the method resides in the non-abelian nature of the algebras generated by the non-local charges. Though the same algebraic structure of the Quantum Group appears in both the QISM and the theory of non-local charges, their physical content is different. One of the primary distinctions of the non-local charges approach is that these charges have no apparent classical formulation. This is due to the fact that their very existence relies on quantum anomalous dimensions of all the fundamental fields ${ }^{1}$.

The QISM is an algebraization of the Bethe-ansatz methods. Though the Bethe-ansatz has had many successes, many of its features (such as the introduction of a pseudo-vacuum and the entailed complicated filling of the Dirac sea) are rather unpleasant for the typical quantum field theorist. In this respect the methods based on non-local charges are more in the usual spirit of quantum field theory.

1 The currents that generate the Yangian symmetry have classical analogs, however their comultiplication is a quantum effect [6] 
Furthermore, these methods are rather general and self-contained, and are well-suited to the clarification of more recent results ${ }^{2}$.

A preliminary version of some of the following results on the SG theory appeared in [15].

\section{Non-Local Charges in 2D QFT}

2a. General Theory. In this section we review the general framework for dealing with non-local charges in two-dimensional quantum field theory. Part of this framework was used in [6].

Due to the possibility of fields with non-trivial braiding relations; quantum field theories in two spacetime dimensions may have non-local conserved currents. The currents, which we denote by $J_{\mu}^{a}(x, t)$, are localized at the space-time points $(x, t)$. Their precise definition (e.g. from a lattice construction or directly in the continuum) requires attaching to the currents a one-dimensional curve going from $-\infty$ to the point $(x, t)$. The precise location of the string attached to the currents is irrelevant except when topological obstructions are encountered. One way to think about this string is in analogy to the disorder line defining disorder fields $[16,17]$. This analogy will be clarified in the sequel. The non-locality of the currents is encoded in their equal-time braiding relations:

$$
J_{\mu}^{a}(x, t) J_{v}^{b}(y, t)=R_{d c}^{a b} J_{v}^{c}(y, t) J_{\mu}^{d}(x, t) ; \text { for } \quad x>y .
$$

The above equation is implicitly time-ordered to the left, e.g. $J_{\mu}^{a}(x, t) J_{v}^{b}(y, t) \equiv$ $J_{\mu}^{a}(x, t+\varepsilon) J_{v}^{b}(y, t)$, for $\varepsilon$ small and positive. The braiding relations (2.1) originate in the topological obstructions encountered while moving the string attached to the currents, and are displayed in Fig. 1. In this figure, time increases upward, and the positions of the strings are dictated by the time-ordering. Associativity of the operator algebra requires the matrix $R_{c d}^{a b}$ to be a solution of the Yang-Baxter equation. A more complete discussion of braiding relations in $2 \mathrm{D}$ quantum field theories can be found in $[18,19]$.

For conserved currents, $\partial_{\mu} J_{\mu}^{a}(x, t)=0$, the global conserved charges $Q^{a}$ acting on the physical Hilbert space are $Q^{a}=\frac{1}{2 \pi i} \int_{t} d x J_{t}^{a}(x, t)$. The charges $\hat{Q}^{a}$ acting on

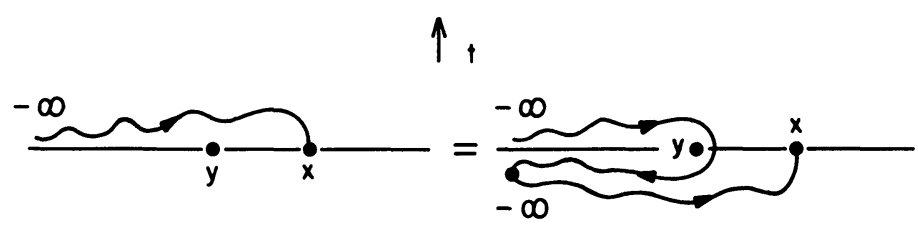

Fig. 1. Graphical representation of braiding

\footnotetext{
${ }^{2}$ We refer the reader to [11] for a unified description of integrable quantum field theories based on symmetries
} 
fields $\Phi^{k}(y)$ are not defined by integrating the currents along an equal-time slice but along a path $\gamma(y)$ from $-\infty$ to $-\infty$ surrounding the point $y$ :

$$
\hat{Q}^{a}\left(\Phi^{k}(y)\right)=\frac{1}{2 \pi i} \int_{\gamma(y)} d z_{\nu} \varepsilon^{v \mu} J_{\mu}^{a}(z) \Phi^{k}(y) .
$$

The contour $\gamma(y)$ is drawn in Fig. 2. There we have drawn the string attached to the currents in the position specified by the order of the fields in (2.2). The exact shape of the contour $\gamma(y)$ is irrelevant due to the conservation of the currents.

The action of the charges on the fields (2.2) can be expressed in terms of generalized commutators, as we now describe. The fields of the theory can be classified into multiplets according to their braiding relations with the currents. We suppose the following braiding relations

$$
J_{\mu}^{a}(x, t) \Phi^{k}(y, t)=\Theta_{b l}^{a k} \Phi^{l}(y, t) J_{\mu}^{b}(x, t) ; \text { for } x>y .
$$

As in (2.1) these braiding relations arise from the obstructions for moving the string attached to the fields. To express (2.2) as generalized braided commutators, let us decompose the contour of integration $\gamma(y)$ into the difference of two contours $\gamma_{+}$and $\gamma_{-}, \gamma(y)=\gamma_{+}-\gamma_{-}$, as in Fig. 3. Integrating the right-hand side of (2.2) along the contour $\gamma_{+}$gives the product $Q^{a} \Phi^{k}(y)$. When the currents are localized on the curve $\gamma_{-}$, the braiding relations (2.3) can be used to move the string through the point $y$, giving the product $\Theta_{b l}^{a k} \Phi^{l}(y) Q^{b}$. We gather everything into the result:

$$
\hat{Q}^{a}\left(\Phi^{k}(y)\right)=Q^{a} \Phi^{k}(y)-\Theta_{b l}^{a k} \Phi^{l}(y) Q^{b} .
$$

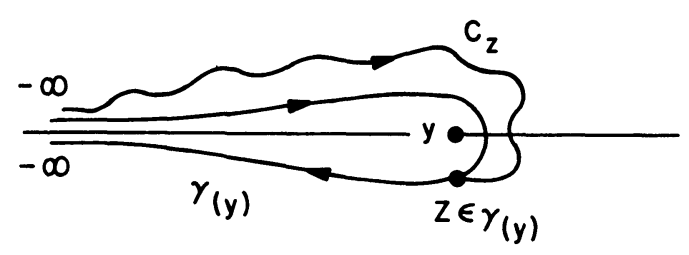

Fig. 2. The contour of integration for the action of non-local charges on fields. The curve $C_{z}$ denotes the string

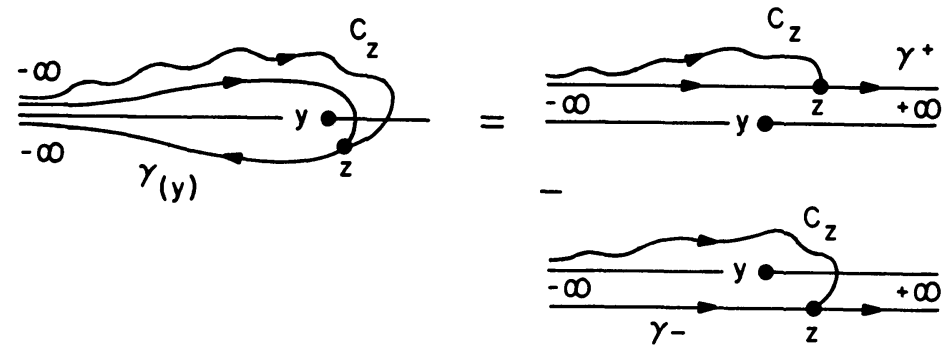

Fig. 3. Decomposition of the contour of Fig. 2 
In particular if we consider $\Phi(y)$ as the time-component of the currents, (2.4) shows that the global conserved charges $Q^{a}$ satisfying braided commutation relations:

$$
Q^{a} Q^{b}-R_{d c}^{a b} Q^{c} Q^{d}=\hat{Q}^{a}\left(Q^{b}\right)
$$

Next we consider the action of the charges on a product of fields. This will define for us the comultiplication $\Delta\left(Q^{a}\right)$ of the charges. For simplicity, consider the action on a product of two fields. This is defined as

$$
\hat{Q}^{a}\left(\Phi^{k}\left(y_{1}\right) \Phi^{n}\left(y_{2}\right)\right)=\frac{1}{2 \pi i} \int_{\gamma_{12}} d z_{v} \varepsilon^{v \mu} J_{\mu}^{a}(z) \Phi^{k}\left(y_{1}\right) \Phi^{n}\left(y_{2}\right) .
$$

The contour $\gamma_{12}$ encloses both space-time points $y_{1}$ and $y_{2}$. See Fig. 4 . The contour $\gamma_{12}$ can be decomposed into the sum of two contours, $\gamma_{12}=\gamma_{1}+\gamma_{2}$, as in Fig. 4 . The integration over the contour $\gamma_{1}$ gives the action of the charges on $\Phi^{k}\left(y_{1}\right)$. After having taken into account the braiding relations between the currents and the fields $\Phi^{k}\left(y_{1}\right)$, the integration over the contour $\gamma_{2}$ gives the action of the charges on $\Phi^{n}\left(y_{2}\right)$. Thus we obtain:

$$
\hat{Q}^{a}\left(\Phi^{k}\left(y_{1}\right) \Phi^{n}\left(y_{2}\right)\right)=\hat{Q}^{a}\left(\Phi^{k}\left(y_{1}\right)\right) \Phi^{n}\left(y_{2}\right)+\left(\Theta_{b l}^{a k} \Phi^{l}\left(y_{1}\right)\right) \hat{Q}^{b}\left(\Phi^{n}\left(y_{2}\right)\right) .
$$

Let us arrange the quantum numbers of the fields $\Phi\left(y_{1}\right)\left(\Phi\left(y_{2}\right)\right)$ into a vector space $V_{1}\left(V_{2}\right)$. The action of the charges $\hat{Q}^{a}$ on the product of two fields is then an operator on $V_{1} \otimes V_{2}$, which is denoted by $\Delta\left(\hat{Q}^{a}\right)$, and defines the comultiplication. In this compact notation (2.7) becomes

$$
\Delta\left(\hat{Q}^{a}\right)=\hat{Q}^{a} \otimes 1+\Theta_{b}^{a} \otimes \hat{Q}^{b},
$$

where $\Theta_{b}^{a}$ is the braiding operator and is a matrix acting on the vector space $V_{1}$. More specifically, $\left\langle l\left|\Theta_{b}^{a}\right| k\right\rangle=\Theta_{b l}^{a k}$. The same comultiplication holds for the global charges $Q^{a}$.

A lattice formulation can also be developed for the above continuum results. The lattice approach reveals that non-local conserved currents are consequences of quantum group invariance of the local hamiltonian. On the lattice, the non-local
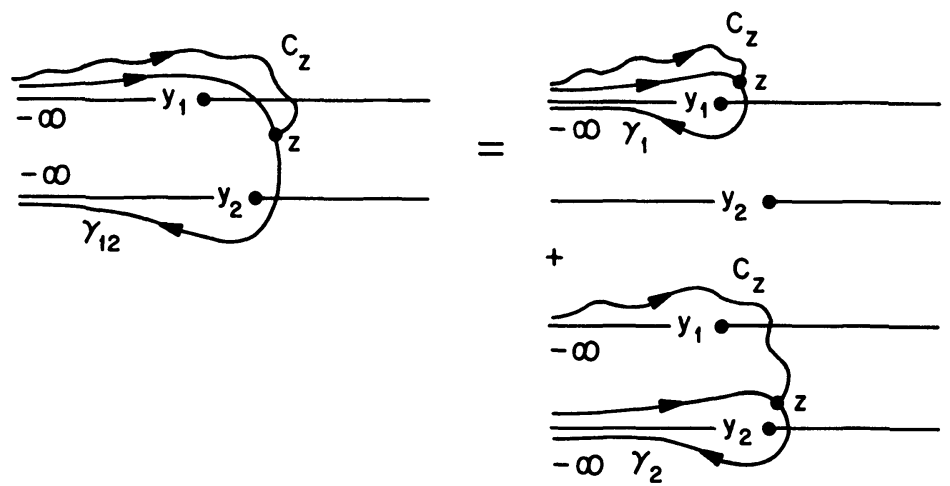

Fig. 4. The contour defining the action of non-local charges on a product of two fields 
currents act as quantum Lie derivatives. This shows that non-commutative differential calculus [20-22] is the geometrical tool appropriate for describing non-local symmetries in two-dimensional quantum field theories. The lattice formulation will be described elsewhere [23].

2b. The Algebra of Charges in Perturbed CFT. In this section we describe the commutation relations of non-local conserved charges in perturbed conformally invariant field theory (CFT).

Consider a conformal field theory perturbed by a relevant operator with zero Lorentz spin. The perturbing field can be represented by $\Phi_{\text {pert. }}(z, \bar{z})=\phi_{\text {pert. }}(z) \bar{\phi}_{\text {pert. }}(\bar{z})$. The Euclidean action is taken to be

$$
S=S_{\mathrm{CFT}}+\frac{\lambda}{2 \pi} \int d^{2} z \Phi_{\text {pert. }}(z, \bar{z})
$$

where $\lambda$ is a generally dimensionful parameter that measures the strength of the perturbation away from the conformal limit. Here $z$ and $\bar{z}$ denote Euclidean coordinates $^{3}$. Chiral fields $F(z, \bar{z}), \bar{F}(z, \bar{z})$ satisfy $\partial_{\bar{z}} F(z, \bar{z})=\partial_{z} \bar{F}(z, \bar{z})=0$ in the conformal limit. Equations of motion for the perturbed chiral fields which are local with respect to the perturbing field can be deduced to first order in perturbation theory using Zamolodchikov's approach [24]:

$$
\begin{aligned}
& \partial_{\bar{z}} F(z, \bar{z})=\lambda \oint_{z} \frac{d w}{2 \pi i} \Phi_{\text {pert. }}(w, \bar{z}) F(z), \\
& \partial_{z} \bar{F}(z, \bar{z})=\lambda \oint_{\bar{z}} \frac{d \bar{w}}{2 \pi i} \Phi_{\text {pert. }}(z, \bar{w}) \bar{F}(\bar{z}) .
\end{aligned}
$$

Equations of motion to first order can be exact to all orders in perturbation theory, as we will see.

Let us now suppose that there are currents conserved to first order in perturbation theory:

$$
\begin{aligned}
& \partial_{\bar{z}} J^{a}(z, \bar{z})=\partial_{z} H^{a}(z, \bar{z}), \\
& \partial_{z} \bar{J}^{\bar{a}}(z, \bar{z})=\partial_{\bar{z}} \bar{H}^{\bar{a}}(z, \bar{z}) .
\end{aligned}
$$

We assume that in the conformal theory these currents are chiral fields; i.e. when $\lambda=0$ they satisfy $\partial_{\bar{z}} J^{a}=\partial_{z} \bar{J}^{\bar{a}}=0$. We also suppose that they are local with respect to the perturbing field. The condition for the currents to be conserved to first order in perturbation theory is then a condition on the residue of the operator product expansion (OPE) between them and the perturbing field. Namely, the conservation laws (2.11) hold if the residues of these OPE's are total derivatives:

$$
\begin{aligned}
& \operatorname{Res}_{z=w}\left(\phi_{\text {pert. }}(w) J^{a}(z)\right)=\partial_{z} h^{a}(z), \\
& \operatorname{Res}_{\bar{z}=\bar{w}}\left(\bar{\phi}_{\text {pert. }}(\bar{w}) \bar{J}^{\bar{a}}(\bar{z})\right)=\partial_{\bar{z}} \bar{h}^{\bar{a}}(\bar{z}) .
\end{aligned}
$$

\footnotetext{
${ }^{3}$ We will always denote Euclidean light-cone coordinates as $z$ and $\bar{z}$, whereas $x, t$ and $x^{ \pm}=(x \pm t) / 2$ will signify Minkowski coordinates. Their relation is $z=i x^{+}=\left(t^{E}+i x\right) / 2$, $\bar{z}=-i x^{-}=\left(t^{E}-i x\right) / 2$, where $t^{E}=i t$. We follow the convention $d^{2} z \equiv i d z d \bar{z}=-d x d t^{E} / 2$
} 
The conditions (2.12) follow from Zamolodchikov's equation of motion (2.10). In (2.11) the fields $H^{a}$ are then

$$
H^{a}(z, \bar{z})=\lambda h^{a}(z) \bar{\phi}_{\text {pert. }}(\bar{z})
$$

and similarly for $\bar{H}^{\bar{a}}(z, \bar{z})$. From the conserved currents (2.11) we define the conserved charges,

$$
\begin{aligned}
& Q^{a}=\frac{1}{2 \pi i}\left(\int d z J^{a}+\int d \bar{z} H^{a}\right), \\
& \bar{Q}^{\bar{a}}=\frac{1}{2 \pi i}\left(\int d \bar{z} \bar{J}^{\bar{a}}+\int d z \bar{H}^{\bar{a}}\right) .
\end{aligned}
$$

Since the currents $J^{a}$ and $\bar{J}^{\bar{a}}$ can be non-local, we allow for non-trivial braiding between them:

$$
J^{a}(x, t) \bar{J}^{\bar{a}}(y, t)=R_{b \bar{b}}^{a \bar{J}} \bar{J}^{\bar{b}}(y, t) J^{b}(x, t) ; \quad \forall x, y .
$$

There is no contradiction in having the above braiding relations defined for all $x, y$; we will give explicit examples in the sequel. The above relations allow one to defined a time-ordered product $T$ that facilitates the string manipulations of the previous section:

$$
\begin{array}{rlrl}
T\left(J^{a}(x, t+\varepsilon) \bar{J}^{\bar{a}}(y, t)\right) & =J^{a}(x, t+\varepsilon) \bar{J}^{\bar{a}}(y, t) & & \varepsilon>0 \\
& =R_{b \bar{b}}^{a \bar{a}} \bar{J}^{\bar{b}}(y, t) J^{b}(x, t+\bar{\varepsilon}) & \varepsilon<0 .
\end{array}
$$

We suppose that the same braiding relations hold if the $J^{a}\left(\bar{J}^{\bar{a}}\right)$ component of the current is replaced by the corresponding $H^{a}\left(\bar{H}^{\bar{a}}\right)$ component. To find the commutation relations between the charges $Q^{a}$ and $\bar{Q}^{\bar{a}}$ associated to these currents, we apply the general framework explained in the previous section. Using (2.12) it is easy to compute $\hat{Q}^{a}\left(\bar{Q}^{\bar{a}}\right)$ to lowest non-trivial order in perturbation theory. The result is:

$$
Q^{a} \bar{Q}^{\bar{a}}-R_{b \bar{b}}^{a \bar{a}} \bar{Q}^{\bar{b}} Q^{b}=T^{a \bar{a}},
$$

where $T^{a \bar{a}}$ is always a topological charge,

$$
T^{a \bar{a}}=\frac{\lambda}{2 \pi i} \int_{t}\left(d z \partial_{z}+d \bar{z} \partial_{\bar{z}}\right) h^{a}(z) \bar{h}^{\bar{a}}(\bar{z}) .
$$

Equation (2.18) may be understood as a generalization of the topological extensions of super-symmetry in two-dimensions [25]. In the following section we will show that in the case of the sine-Gordon theory the relations (2.18) are those of the quantum $s l(2)$ loop algebra.

\section{Non-Local Charges in the Sine-Gordon Theory}

In this section we describe the non-local charges that characterize the sine-Gordon theory. We will show that these charges generate the quantum $s l(2)$ loop algebra. Our analysis provides a new derivation of the soliton $S$-matrix. 
3a. A Review of the Sine-Gordon Theory. The quantum sine-Gordon theory is described by the Euclidean action

$$
S=\frac{1}{4 \pi} \int d^{2} z \partial_{z} \Phi \partial_{\bar{z}} \Phi+\frac{\lambda}{\pi} \int d^{2} z: \cos (\hat{\beta} \Phi): .
$$

The parameter $\hat{\beta}$ is a coupling constant; it is related to the conventionally normalized coupling by $\hat{\beta}=\beta / \sqrt{4 \pi}$ (see ref. [9]). The values of the coupling $\hat{\beta}=1$ and $\hat{\beta}=\sqrt{2}$ are known to correspond to a free Dirac fermion and to the $S U(2)$ Gross-Neveu model respectively. The parameter $\lambda$ defines the mass scale of the model; in the deep ultra-violet it is zero. For $\hat{\beta} \leqq \sqrt{2}$ the action can be renormalized by normal-ordering the $\cos (\hat{\beta} \Phi)$ interaction and absorbing the infinities into $\lambda$; the coupling constant $\hat{\beta}$ is thereby unrenormalized [9]. With the above normalization of the kinetic term the Euclidean propagator is:

$$
\langle\Phi(z, \bar{z}) \Phi(0,0)\rangle=-\log (z \bar{z}) .
$$

We will treat the action (3.1) as a perturbation of a conformal theory in the sense developed by Zamolodchikov [24]. Namely, we treat the $\lambda \cos (\hat{\beta} \Phi)$ term as a perturbation of the conformal field theory corresponding to a single free boson. Recognizing that the $\cos (\hat{\beta} \Phi)$ potential imposes the periodicity $\widehat{\beta} \Phi \equiv \widehat{\beta} \Phi+2 \pi$, one sees that the free boson is compactified on a circle of radius $R=1 / \hat{\beta}$, where the duality is $R \rightarrow \frac{1}{2 R}$ (see e.g. [26]). For $\hat{\beta}<\sqrt{2}$, the perturbing field is relevant: its (holomorphic, anti-holomorphic) anomalous dimensions are less than one. This implies that in the deep ultraviolet, the theory is conformal. Following Zamolodchikov [24], this allows us to assume that the space of fields has not been drastically modified by the perturbation. In particular it allows us to suppose that all the operators $\mathcal{O}(x, t)$ of the sine-Gordon theory have a smooth ultra-violet limit and that they are in correspondence with the fields of the ultra-violet CFT. We can thus label in a unique way the fields of the sine-Gordon theory by the corresponding fields in the ultra-violet limit. In the massless limit, the free boson can be expanded as $\Phi(z, \bar{z})=\phi(z)+\bar{\phi}(\bar{z})$ with $\langle\phi(w) \phi(z)\rangle=-\log (w-z)$ and similarly for $\bar{\phi}$. The fields of the ultra-violet CFT are products of the chiral vertex operators $\exp (i \alpha \phi(z))$ and $\exp (i \alpha \bar{\phi}(\bar{z}))$ and of their Virasoro descendents. The anomalous (holomorphic, antiholomorphic) dimensions $(\Delta, \bar{\Delta})$ of these exponential operators are:

$$
\Delta\left(\exp (i \alpha \phi(z))=\bar{\Delta}(\exp (i \alpha \bar{\phi}(\bar{z})))=\frac{\alpha^{2}}{2} .\right.
$$

The perturbing operator $\cos (\hat{\beta} \Phi)$ is thus relevant for $\hat{\beta}<\sqrt{2}$ as indicated above.

In the deep ultra-violet limit the (anti)-chiral components $\phi(x, t)$ and $\bar{\phi}(x, t)$ can be expressed in a non-local way in terms of the sine-Gordon field $\Phi(x, t)$. The relations are:

$$
\begin{aligned}
& \phi(x, t)=\frac{1}{2}\left(\Phi(x, t)+\int_{-\infty}^{x} d y \partial_{t} \Phi(y, t)\right) \\
& \bar{\phi}(x, t)=\frac{1}{2}\left(\Phi(x, t)-\int_{-\infty}^{x} d y \partial_{t} \Phi(y, t)\right) .
\end{aligned}
$$


Equations (3.4) are proved by integrating the following equality, $\left(\partial_{x}+\partial_{t}\right) \Phi=$ $\left(\partial_{x}+\partial_{t}\right) \phi=2 \partial_{x} \phi$, which holds in the massless limit, because there we have $\left(\partial_{x}-\partial_{t}\right) \phi=\left(\partial_{x}+\partial_{t}\right) \bar{\phi}=0$. A similar computation yields the expression for the anti-holomorphic component. Though the above non-local expressions (3.4) were derived in the massless limit, we can take them to define the chiral components $\phi$ and $\bar{\phi}$ in exponential operators in the massive theory also, because of the correspondence mentioned above.

Let us illustrate the perturbative approach by deriving the equation of motion for the sine-Gordon field. The Zamolodchikov equations of motion (2.10) for the chiral fields $i \partial_{z} \phi$ and $i \partial_{\bar{z}} \bar{\phi}$, together with the operator product expansion (OPE),

$$
i \partial_{z} \phi(z) \exp (i \alpha \phi(w)) \sim \frac{\alpha}{z-w} \exp (i \alpha \phi(w))+\cdots
$$

give to first order in $\lambda$ :

$$
\partial_{z} \partial_{\bar{z}} \Phi(z, \bar{z})=-2 \lambda \hat{\beta} \sin (\hat{\beta} \Phi(z, \bar{z})) .
$$

Therefore, for the sine-Gordon field $\Phi$, the equation of motion is exact to first order in perturbation. For relevant perturbations, because the coupling constant $\lambda$ has positive dimension, local equations of motion are exact to a finite order in perturbation theory. In. the case of the sine-Gordon theory, for the field $i \partial_{z} \phi$ it is easy to see by scaling arguments that only the first order appears in the equation of motion.

The sine-Gordon theory has a well known topological current:

$$
\mathscr{I}^{\mu}(x, t)=\frac{\hat{\beta}}{2 \pi} \varepsilon^{\mu \nu} \partial_{v} \Phi(x, t),
$$

where $\varepsilon^{\mu v}=-\varepsilon^{v \mu}$. We take the convention $\varepsilon^{01}=1$. The topological charge is:

$$
\mathscr{T}=\frac{\widehat{\beta}}{2 \pi} \int_{-\infty}^{+\infty} d x \partial_{x} \Phi=\frac{\widehat{\beta}}{2 \pi}(\Phi(x=\infty)-\Phi(x=-\infty)) .
$$

The normalization of the topological current is fixed by the periodicity of the $\cos (\hat{\beta} \Phi)$ potential. More specifically, the topological solitons that correspond to single particles in the quantum theory are described classically by field configurations with $\mathscr{T}= \pm 1$. These solitons are kinks that connect two neighboring vacua in the $\cos (\hat{\hat{\beta}} \Phi)$ potential. In the quantum theory the topological charge $\mathscr{T}(\mathcal{O})$ of an operator $\mathcal{O}$ is defined by the commutation relation $[\mathscr{T}, \mathcal{O}]=\mathscr{T}(\mathcal{O}) \mathcal{O}$. The topological charge of the vertex operators is thus:

$$
\mathscr{T}(\exp (i \alpha \phi+i \bar{\alpha} \bar{\phi}))=\hat{\beta}(\alpha-\bar{\alpha}) .
$$

3b. The Non-Local Conserved Charges. In this section we derive the existence of conserved currents generated by fields of the form $J_{\alpha}=\exp (i \alpha \phi)$ or $\bar{J}_{\alpha}=\exp (i \alpha \bar{\phi})$.

As explained in Sect. $2 b$, the existence of a conserved current in the perturbed theory is the requirement (2.12) on the OPE of the current $J_{\alpha}\left(\right.$ or $\bar{J}_{\alpha}$ ) with the perturbing field. The perturbing field is $\Phi_{\text {pert. }}=\exp (i \hat{\beta} \Phi)+\exp (-i \hat{\beta} \Phi)$. Using the OPE

$$
\exp (i \alpha \phi(z)) \exp (i \hat{\beta} \phi(w)) \sim(z-w)^{\alpha \hat{\beta}} \exp (i(\alpha+\hat{\beta}) \phi(w))+\cdots
$$


(and similarly for the anti-holomorphic sector) we see that the condition (2.12) for a conservation law for $J_{\alpha}$ amounts to a condition on $\alpha$; namely $\alpha= \pm 2 / \hat{\beta}$. Therefore we find the following conserved currents:

$$
\partial_{\bar{z}} J_{ \pm}=\partial_{z} H_{ \pm} ; \quad \partial_{z} \bar{J}_{ \pm}=\partial_{\bar{z}} \bar{H}_{ \pm}
$$

where

$$
\begin{aligned}
J_{ \pm}(x, t) & =\exp \left( \pm \frac{2 i}{\hat{\beta}} \phi(x, t)\right)=\exp \left( \pm \frac{i}{\hat{\beta}} \Phi(x, t) \pm \frac{i}{\hat{\beta}} \int_{-\infty}^{x} d y \partial_{t} \Phi\right) \\
H_{ \pm}(x, t) & =\lambda \frac{\hat{\beta}^{2}}{\hat{\beta}^{2}-2} \exp \left[ \pm i\left(\frac{2}{\hat{\beta}}-\hat{\beta}\right) \phi(x, t) \mp i \hat{\beta} \bar{\phi}(x, t)\right] \\
& =\lambda \frac{\hat{\beta}^{2}}{\hat{\beta}^{2}-2} \exp \left[ \pm i\left(\frac{1}{\hat{\beta}}-\hat{\beta}\right) \Phi(x, t) \pm \frac{i}{\hat{\beta}} \int_{-\infty}^{x} d y \partial_{t} \Phi\right] \\
\bar{J}_{ \pm}(x, t) & =\exp \left(\mp \frac{2 i}{\hat{\beta}} \bar{\phi}(x, t)\right)=\exp \left(\mp \frac{i}{\hat{\beta}} \Phi(x, t) \pm \frac{i}{\hat{\beta}} \int_{-\infty}^{x} d y \partial_{t} \Phi\right) \\
H_{ \pm}(x, t) & =\lambda \frac{\hat{\beta}^{2}}{\hat{\beta}^{2}-2} \exp \left[\mp i\left(\frac{2}{\hat{\beta}}-\hat{\beta}\right) \bar{\phi}(x, t) \pm i \hat{\beta} \phi(x, t)\right] \\
& =\lambda \frac{\hat{\beta}^{2}}{\hat{\beta}^{2}-2} \exp \left[\mp i\left(\frac{1}{\hat{\beta}}-\hat{\beta}\right) \Phi(x, t) \pm \frac{i}{\hat{\beta}} \int_{-\infty}^{x} d y \partial_{t} \Phi\right]
\end{aligned}
$$

By simple scaling arguments, it easy to check that for $\hat{\beta}^{2}$ irrational, the equations of motion for $J_{ \pm}$and $\bar{J}_{ \pm}$are exact to first order in perturbation theory. Therefore the currents (3.12) are conserved to all orders. From these conserved currents we define four conserved charges:

$$
\begin{aligned}
& Q_{ \pm}=\frac{1}{2 \pi i}\left(\int d z J_{ \pm}+\int d \bar{z} H_{ \pm}\right), \\
& \bar{Q}_{ \pm}=\frac{1}{2 \pi i}\left(\int d \bar{z} \bar{J}_{ \pm}+\int d z \bar{H}_{ \pm}\right) .
\end{aligned}
$$

The Lorentz spin $s$ of the currents $J_{ \pm}\left(\bar{J}_{ \pm}\right)$is $s=\frac{2}{\hat{\beta}^{2}}\left(-\frac{2}{\hat{\beta}^{2}}\right)$; this follows from their anomalous scaling dimensions and the relation $s=\Delta-\bar{\Delta}$. The Lorentz spin of the conserved charges is thus:

$$
\frac{1}{\gamma} \equiv \operatorname{spin}\left(Q_{ \pm}\right)=-\operatorname{spin}\left(\bar{Q}_{ \pm}\right)=\frac{2}{\hat{\beta}^{2}}-1
$$

The conserved charges (3.13) are non-local due to the fact that the (anti)-chiral components, $\phi$ and $\bar{\phi}$, of the sine-Gordon field $\Phi$ are non-local. This non-locality is reflected in the relations (3.4). The non-local expressions (3.4) manifest the strings attached to the currents that were referred to in Sect. 2. The braiding relations arising from the non-locality are independent of the scale; thus they can be described in the ultra-violet limit without loss of information. 
3c. The Algebra of Non-Local Charges. We now use the results of Sect. 2 to determine the algebra obeyed by the non-local charges (3.13). This requires knowledge of the braiding properties of the non-local currents (3.12). These follow from the expressions (3.4) for the chiral components $\phi$ and $\bar{\phi}$ of the sine-Gordon field $\Phi$, and from the canonical commutation relations of $\Phi$ :

$$
\left[\Phi(x, t), \partial_{t} \Phi(x, t)\right]=4 \pi i \delta(x-y) \text {. }
$$

Using the fact that $e^{A} e^{B}=e^{[A, B]} e^{B} e^{A}$ for $[A, B]$ a $c$-number, we deduce:

$\exp (i a \phi(x, t)) \exp (i b \phi(y, t))=e^{ \pm i \pi a b} \exp (i b \phi(y, t)) \exp (i a \phi(x, t)) ;$ for $\quad x \gtrless y$,

$\exp (i a \bar{\phi}(x, t)) \exp (i b \bar{\phi}(y, t))=e^{\mp i \pi a b} \exp (i b \bar{\phi}(y, t)) \exp (i a \bar{\phi}(x, t)) ;$ for $\quad x \gtrless y$,

$\exp (i a \phi(x, t)) \exp (i b \bar{\phi}(y, t))=e^{i \pi a b} \exp (i b \bar{\phi}(y, t)) \exp (i a \phi(x, t)) ; \quad \forall x, y$.

These relations (3.16) are the braiding relations for generalized parafermions $[16,17]$ for the group $\alpha Z$. In making this analogy, the disorder fields are identified with $\mu_{a}(x, t)=\exp \left(\frac{i a}{2} \int_{-\infty}^{x} d y \partial_{t} \Phi(y, t)\right)$ in the ultra-violet limit, and exhibit the strings. This connection is further clarified in Appendix D.

For the currents of interest (3.12), the braiding relations (3.16) imply:

$$
\begin{array}{ll}
J_{ \pm}(x, t) \bar{J}_{\mp}(y, t)=q^{-2} \bar{J}_{\mp}(y, t) J_{ \pm}(x, t) ; & \forall x, y, \\
J_{ \pm}(x, t) \bar{J}_{ \pm}(y, t)=q^{2} \bar{J}_{ \pm}(y, t) J_{ \pm}(x, t) ; & \forall x, y,
\end{array}
$$

where

$$
q=\exp \left(-2 \pi i / \hat{\beta}^{2}\right)=-\exp (-i \pi / \gamma)
$$

The same braiding relations hold if we replace any of the fields $J_{ \pm}\left(\bar{J}_{ \pm}\right)$by the corresponding field $H_{ \pm}\left(\bar{H}_{ \pm}\right)$.

Using the above braiding relations Eqs. (2.17) and (2.18) become:

$$
\begin{aligned}
Q_{ \pm} \bar{Q}_{ \pm}-q^{2} \bar{Q}_{ \pm} Q_{ \pm} & =0 \\
Q_{ \pm} \bar{Q}_{\mp}-q^{-2} \bar{Q}_{\mp} Q_{ \pm} & =\frac{\lambda}{2 \pi i} \gamma^{2} \int_{t} d x \partial_{x}\left[\exp \left( \pm i\left(\frac{2}{\hat{\beta}}-\hat{\beta}\right) \Phi(x, t)\right)\right] .
\end{aligned}
$$

The topological charges on the right-hand side of (3.19) can be expressed in terms of the usual topological charge $\mathscr{T}$ in (3.8). A soliton configuration can be taken to satisfy $\Phi(x=\infty)=0$; the classical soliton solutions do in fact satisfy this. Integrating (3.19) we obtain the algebra to lowest non-trivial order in perturbation theory:

$$
\begin{aligned}
Q_{+} \bar{Q}_{+}-q^{2} \bar{Q}_{+} Q_{+} & =0 \\
Q_{-} \bar{Q}_{-}-q^{2} \bar{Q}_{-} Q_{-} & =0 \\
Q_{+} \bar{Q}_{-}-q^{-2} \bar{Q}_{-} Q_{+} & =a\left(1-q^{2 \mathscr{T}}\right), \\
Q_{-} \bar{Q}_{+}-q^{-2} \bar{Q}_{+} Q_{-} & =a\left(1-q^{-2 \mathscr{T}}\right),
\end{aligned}
$$




$$
\begin{aligned}
& {\left[\mathscr{T}, Q_{ \pm}\right]= \pm 2 Q_{ \pm},} \\
& {\left[\mathscr{T}, \bar{Q}_{ \pm}\right]= \pm 2 \bar{Q}_{ \pm},}
\end{aligned}
$$

where $a \equiv \lambda \gamma^{2} / 2 \pi i$. In deriving the last equations we have used (3.9).

It is possible to show by scaling arguments that the algebra (3.20) is exact to all orders in perturbation theory. Thus the algebra (3.20) can be taken as a non-perturbative definition of the theory. The scaling argument goes as follows. The scaling dimension of an operator $\mathcal{O}$ is $\operatorname{dim}(\mathcal{O})=\Delta(\mathcal{O})+\bar{\Delta}(\mathcal{O})$. The most general possibility for the right-hand side of $(3.19 \mathrm{~b})$ is $\sum_{n} \lambda^{n}\left(\int d z \mathcal{O}_{n}+\int d \bar{z} \overline{\mathcal{O}}_{n}\right)$. From the fact that the dimension of the left-hand side of $(3.19 \mathrm{~b})$ is $2 / \gamma$, and $\operatorname{dim}(\lambda)=2-\hat{\beta}^{2}$, one finds that $\operatorname{dim}\left(\mathcal{O}_{n}\right)-1=4 / \widehat{\beta}^{2}+n \widehat{\beta}^{2}-2(1+n)$. This equation can only be satisfied if $\mathcal{O}_{n}=\partial_{z} H_{n}, \overline{\mathcal{O}}_{n}=\partial_{\bar{z}} \bar{H}_{n}$. The field $H_{n}$ must be a product of $\exp \left( \pm \frac{2}{\hat{\beta}} \Phi\right)$ with the perturbing field raised to some power $k$. Therefore $H_{n} \sim \exp \left( \pm\left(\frac{2}{\hat{\beta}}+k \hat{\beta}\right) \Phi\right), k \in Z$, and $\operatorname{dim}\left(\mathcal{O}_{n}\right)-1=\left(\frac{2}{\hat{\beta}}+k \hat{\beta}\right)^{2}$. Assuming that $\hat{\beta}$ is irrational, one thus finds that $k^{2}=n$ and $2 k=-1-n$. The only solution is $n=1$.

The algebra (3.20) is a known infinite dimensional algebra, namely the $q$-deformation of the $s l(2)$ affine Kac-Moody algebra, denoted $\widehat{s l} l_{q}(2)$, with zero center $[2,3]$. Only the Serre relations for $\widehat{s l_{q}(2)}$ are missing in (3.20). This algebra is reviewed in Appendix A. Let $E_{i}, F_{i}, H_{i}, i=0,1$, denote the Chevaley basis for the centerless $\widehat{s l}(2)$ algebra in the principal gradation (see Appendix A for definitions). They satisfy the following defining relations:

$$
\begin{aligned}
& {\left[H_{i}, E_{j}\right]=a_{i j} E_{j},} \\
& {\left[H_{i}, F_{j}\right]=-a_{i j} F_{j},} \\
& {\left[E_{i}, F_{j}\right]=\delta_{i j} \frac{q^{H_{i}}-q^{-H_{i}}}{q-q^{-1}},}
\end{aligned}
$$

with $a_{i j}$ the Cartan matrix of the affine Kac-Moody algebra $\widehat{s l(2)}$. The relations between the non-local charges $Q_{ \pm}$and $\bar{Q}_{ \pm}$and these generators are:

$$
\begin{aligned}
Q_{+} & =c E_{1} q^{H_{1} / 2} ; \quad Q_{-}=c E_{0} q^{H_{0} / 2} ; \\
\bar{Q}_{-} & =c F_{1} q^{H_{1} / 2} ; \quad \bar{Q}_{+}=c F_{0} q^{H_{0} / 2} ; \\
\mathscr{T} & =H_{1}=-H_{0},
\end{aligned}
$$

where $c$ is a constant $\left(c^{2}=\frac{\lambda}{2 \pi i} \gamma^{2}\left(q^{-2}-1\right)\right)$. The last equation in (3.14) reflects the fact that the center of $\widehat{s l}(2)$ is zero.

Note that when $q=-i,(\hat{\beta}=2 / \sqrt{3})$, the algebra (3.20) is a topological extension of the $N=2$ supersymmetry algebra. This fact was recently used in the study of perturbations of the $N=2$ superconformal series [15].

3d. The Fundamental Soliton Fields. We will now construct the fundamental quantum fields that create sine-Gordon solitons out of the vacuum. These fields 
must satisfy a number of requirements: (i) they must have topological charge \pm 1 ; (ii) they must transform in a well defined way with respect to the non-local charges. It turns out that these requirements are not independent. The condition (ii) is fundamental for consistency of our approach based on non-local charges. Moreover, as we will see, these requirements imply that the soliton fields have well defined equations of motions.

There are large families of operators with topological charge \pm 1 . These operators differ by a product with local fields, and in general differ in Lorentz spin. Among them, there are four fields which generate these families. These fields, which we call the fundamental soliton fields, are defined by:

$$
\begin{aligned}
& \Psi_{ \pm}(x, t)=\exp \left( \pm \frac{i}{\hat{\beta}} \phi(x, t)\right) \\
& \bar{\Psi}_{ \pm}(x, t)=\exp \left(\mp \frac{i}{\hat{\beta}} \bar{\phi}(x, t)\right) .
\end{aligned}
$$

They have topological charges \pm 1 as can be seen from (3.9). They are chosen for the special properties of their OPE's with the currents (3.19). Namely,

$$
\begin{aligned}
& J_{+}(z) \Psi_{-}(w) \sim \frac{1}{(z-w)^{2 / \hat{\beta}^{2}}} \Psi_{+}(w)+\cdots \\
& J_{-}(z) \Psi_{+}(w) \sim \frac{1}{(z-w)^{2 / \hat{\beta}^{2}}} \Psi_{-}(w)+\cdots
\end{aligned}
$$

The relations (3.24) imply that the non-local charges will transform solitons into anti-solitons and vise versa. Thereby, the soliton states will form a representation of the algebra of non-local charges. The solitons fields in (3.20) are also characterized by non-trivial Loretnz spin:

$$
\operatorname{spin}\left(\Psi_{ \pm}\right)=-\operatorname{spin}\left(\bar{\Psi}_{ \pm}\right)=\frac{1}{2 \widehat{\beta}^{2}} .
$$

It is interesting to display explicitly the braiding relations satisfied by the soliton fields (3.23). They can be derived from the relations (3.16). Let $\Psi_{\mathscr{T}= \pm 1}$ and $\bar{\Psi}_{\mathscr{T}= \pm 1}$ represent the soliton fields. Then,

$$
\begin{array}{ll}
\boldsymbol{\Psi}_{\mathscr{T}}(x, t) \Psi_{\mathscr{T}}(y, t)=q^{\mp \mathscr{T}^{\prime} / 2} \Psi_{\mathscr{T}}(y, t) \Psi_{\mathscr{T}}(x, t) ; & \text { for } \quad x \gtrless y, \\
\overline{\boldsymbol{\Psi}}_{\mathscr{T}}(x, t) \bar{\Psi}_{\mathscr{T}^{\prime}}(y, t)=q^{ \pm \mathscr{T}^{\prime} / 2} \bar{\Psi}_{\mathscr{T}}(y, t) \bar{\Psi}_{\mathscr{T}}(x, t) ; & \text { for } \quad x \gtrless y, \\
\boldsymbol{\Psi}_{\mathscr{T}}(x, t) \bar{\Psi}_{\mathscr{T}^{\prime}}(y, t)=q^{\mathscr{T} \mathscr{T}^{\prime} / 2} \bar{\Psi}_{\mathscr{T}^{\prime}}(y, t) \Psi_{\mathscr{T}}(x, t) ; & \forall x, y .
\end{array}
$$

These relations are the braiding relations for the parafermions considered by Smirnov [27]. However his approach is different from ours in that his starting point is the construction of form factors from the knowledge of the sine-Gordon $S$-matrix, whereas we obtain these relations from (3.4) and (3.23), and will derive the $S$-matrix in the following section.

Let us now study the equations of motion for the soliton fields. The soliton fields are chiral or anti-chiral in the ultra-violet limit. They are local with respect to the perturbing field. This relative locality is a consequence of the fact that the 
soliton fields have topological charge \pm 1 . Therefore their equations of motion can be analyzed using (2.10). One finds:

$$
\begin{aligned}
& \partial_{\bar{z}} \Psi_{ \pm}=\lambda \exp \left[\mp i \hat{\beta} \bar{\phi} \mp i\left(\hat{\beta}-\frac{1}{\hat{\beta}}\right) \phi\right], \\
& \partial_{z} \bar{\Psi}_{ \pm}=\lambda \exp \left[ \pm i \hat{\beta} \phi \pm i\left(\hat{\beta}-\frac{1}{\hat{\beta}}\right) \bar{\phi}\right] .
\end{aligned}
$$

The equations of motion for the soliton fields cannot be expressed solely in terms of the soliton fields themselves. This leads us to define some auxiliary chiral and anti-chiral fields,

$$
\begin{aligned}
& \chi_{ \pm}(x, t)=\exp \left(\mp i\left(\hat{\beta}-\frac{1}{\hat{\beta}}\right) \phi(x, t)\right), \\
& \bar{\chi}_{ \pm}(x, t)=\exp \left( \pm i\left(\hat{\beta}-\frac{1}{\hat{\beta}}\right) \bar{\phi}(x, t)\right) .
\end{aligned}
$$

The equations of motion now become:

$$
\begin{aligned}
& \partial_{\bar{z}} \Psi_{ \pm}=\lambda:\left(\bar{\chi}_{\mp} \bar{\Psi}_{ \pm}\right) \chi_{ \pm}: \\
& \partial_{z} \bar{\Psi}_{ \pm}=\lambda:\left(\chi_{\mp} \Psi_{ \pm}\right) \bar{\chi}_{ \pm}:
\end{aligned}
$$

where the normal ordering is defined by a point-splitting procedure. The fields $\left(\Psi_{ \pm}, \bar{\Psi}_{ \pm}\right)$and $\left(\chi_{ \pm}, \bar{\chi}_{ \pm}\right)$are sufficient to describe all the fundamental fields in the theory. The interaction term can be written as

$$
: \cos (\hat{\beta} \Phi):=:\left(\chi_{-} \boldsymbol{\Psi}_{+}\right)\left(\bar{\chi}_{+} \overline{\boldsymbol{\Psi}}_{-}\right):+:\left(\chi_{+} \boldsymbol{\Psi}_{-}\right)\left(\bar{\chi}_{-} \overline{\boldsymbol{\Psi}}_{+}\right): \text {. }
$$

This equation shows the connection between the sine-Gordon model and a deformation of the $S U(2)$ Gross-Neveu models. This connection will be explored in Sect. 3g. The non-local conserved currents (3.19) can also be written in terms of these elementary fields. All these facts attest to the strong internal consistency of the framework based on non-local charges.

3e. The Representation of the Non-Local Charges on Asymptotic Multi-Soliton States. In this section we determine the manner in which the non-local charges (3.13) are represented on asymptotic (in the sense of scattering theory) multi-soliton states.

Let $\left|\alpha= \pm \frac{1}{2}, \theta\right\rangle$ denote a single-soliton state with topological charge $\mathscr{T}=2 \alpha= \pm 1$ and rapidity $\theta$, where $\theta$ parametrizes the on-shell energy-momentum,

$$
E=m \cosh \theta ; \quad P=m \sinh \theta .
$$

We will henceforth set the mass $m$ of the solitons to 1 . An $N$-soliton state is denoted as,

$$
\left|\alpha_{1}, \theta_{1}\right\rangle \otimes\left|\alpha_{2}, \theta_{2}\right\rangle \otimes \cdots \otimes\left|\alpha_{N}, \theta_{N}\right\rangle
$$

The vector space of single soliton states of fixed rapidity will be referred to as $V=$ vect. $\left\{\left|+\frac{1}{2}\right\rangle,\left|-\frac{1}{2}\right\rangle\right\}$.

Consider first the action on single-soliton states. The action of the charges on such states must form a representation of the algebra (3.20). This representation 
can be deduced as follows. We suppose that the fields (3.23) create the solitons. Taking into account the topological charges of the soliton fields, we have the following non-vanishing matrix elements,

$$
\begin{aligned}
& \left\langle 0\left|\Psi_{ \pm}(x, t)\right| \mp 1 / 2, \theta\right\rangle \neq 0, \\
& \left\langle 0\left|\bar{\Psi}_{ \pm}(x, t)\right| \mp 1 / 2, \theta\right\rangle \neq 0,
\end{aligned}
$$

as $t \rightarrow \pm \infty$. Analogous non-vanishing matrix elements exist for any operator having topological charge \pm 1 and which differs from the soliton fields by multiplication with a local operator. Thus we can take either fields of the family generated by the soliton fields $\Psi_{ \pm}$or $\bar{\Psi}_{ \pm}$to create the soliton state $\left| \pm \frac{1}{2}, \theta\right\rangle$ asymptotically. From the OPE's in (3.24) (and similarly for the anti-holomorphic fields) one infers that the charges $Q_{+}$and $\bar{Q}_{+}$will transform anti-solitons to solitons and vise versa for the charges $Q_{-}$and $\bar{Q}_{-}$. More precisely, using the same technique as in Sects. $2 b$ and $3 c$, we derive the exact action of the non-local charges on the soliton fields:

$$
\begin{aligned}
& Q_{ \pm}\left(\bar{\Psi}_{ \pm}\right)=0, \\
& Q_{ \pm}\left(\bar{\Psi}_{\mp}\right)=\lambda: \Psi_{ \pm}\left(\chi_{ \pm} \bar{\chi}_{\mp}\right): \equiv \lambda \hat{\Psi}_{ \pm}, \\
& \bar{Q}_{ \pm}\left(\Psi_{ \pm}\right)=0, \\
& \bar{Q}_{ \pm}\left(\Psi_{\mp}\right)=\lambda: \bar{\Psi}_{ \pm}\left(\bar{\chi}_{ \pm} \chi_{\mp}\right): \equiv \lambda \hat{\bar{\Psi}}_{ \pm} .
\end{aligned}
$$

The fields $\hat{\Psi}_{ \pm}$(or $\hat{\bar{\Psi}}_{ \pm}$), implicitly defined in (3.34), have topological charge \pm 1 . They differ from the fields $\Psi_{ \pm}\left(\right.$or $\overline{\boldsymbol{\Psi}}_{ \pm}$) by the local operators $\chi_{ \pm} \bar{\chi}_{\mp}\left(\right.$ or $\bar{\chi}_{ \pm} \bar{\chi}_{\mp}$ ), and thus also create solitons asymptotically. An easy computation shows that $\operatorname{spin}\left(\hat{\Psi}_{ \pm}\right)=\operatorname{spin}\left(\bar{\Psi}_{\mp}\right)+\frac{1}{\gamma}$. Therefore the action (3.34) is consistent with the Lorentz spin $\pm \frac{1}{\gamma}$ of the non-local charges, as it must be. In rapidity space, a Lorentz boost is represented as a shift of $\theta: \theta \rightarrow \theta-\alpha$. The on-shell operators $\exp ( \pm \theta / \gamma)$ have Lorentz spin $\pm \frac{1}{\gamma}$. Taking all these facts together, we find the following representation of the charges on the asymptotic solitons,

$$
\begin{aligned}
Q_{ \pm} & =c e^{\theta / \gamma} E_{ \pm} q^{ \pm H / 2}, \\
\bar{Q}_{ \pm} & =c e^{-\theta / \gamma} E_{ \pm} q^{\mp H / 2}, \\
\mathscr{T} & =H,
\end{aligned}
$$

where $c$ is a constant, $H=\operatorname{diag}(+1,-1)$ and $E_{ \pm}$are the Pauli spin matrices $\sigma_{ \pm}$.

The comultiplication of the charges defines their action on multi-soliton states. As explained in Sect. $2 \mathrm{a}$, this comultiplication follows from the braiding of the currents with the soliton fields. The required braiding relations are computed from (3.16) using the expressions (3.12) and (3.23). The result is:

$$
\begin{array}{ll}
J_{ \pm}(x, t) \bar{\Psi}_{\mathscr{T}}(y, t)=q^{ \pm \mathscr{T}} \bar{\Psi}_{\mathscr{T}}(y, t) J_{ \pm}(x, t) ; & \forall x, y, \\
\bar{J}_{ \pm}(x, t) \Psi_{\mathscr{T}}(y, t)=q^{\mp \mathscr{T}} \Psi_{\mathscr{T}}(y, t) \bar{J}_{ \pm}(x, t) ; & \forall x, y .
\end{array}
$$

The same braiding relations hold if the field $J_{ \pm}$or $\bar{J}_{ \pm}$is replaced by the corresponding field $H_{ \pm}$or $\bar{H}_{ \pm}$. Therefore, the comultiplication is:

$$
\Delta\left(Q_{ \pm}\right)=Q_{ \pm} \otimes 1+q^{ \pm H} \otimes Q_{ \pm},
$$




$$
\begin{aligned}
\Delta\left(\bar{Q}_{ \pm}\right) & =\bar{Q}_{ \pm} \otimes 1+q^{\mp H} \otimes \bar{Q}_{ \pm}, \\
\Delta(H) & =H \otimes 1+H \otimes 1 .
\end{aligned}
$$

The last relation follows from the additivity of the topological charge $\mathscr{T}$.

It is instructive to compare the above results with the known structure of the $\widehat{s} \widehat{t_{q}(2)}$ loop algebra. (The reader is referred to Appendix A for definitions of the terminology in what follows.) The isomorphism of the representation (3.35) to the $\widehat{s l_{q}(2)}$ representation (3.22) is made explicit by identifying the spectral parameter $x$ in the principal gradation with $\exp (\theta / \gamma)$. The comultiplication (3.37) that we derived in the quantum field theory can be compared with the known comultiplication of $\widehat{s l_{q}(2)}$ using (3.22); they are equivalent as they must be. In particular this implies that the comultiplication provides a representation of the algebra (3.20) on asymptotic states with an arbitrary number of particles. This fact is important in establishing the non-local charges as true symmetries of the theory.

$3 f$. The S-Matrix from the Non-Local Charges. We will now demonstrate how one can use the non-local charges to obtain non-perturbative information about the sine-Gordon theory by providing a derivation of the soliton $S$-matrix.

The integrability of the sine-Gordon theory implies that the set of in-coming and out-going momenta are the same. Let $V_{1} \otimes V_{2}$ denote the Hilbert space of two-soliton states of fixed rapidities, i.e. $V_{1} \otimes V_{2}$ is spanned by the states $\left|\alpha_{1}= \pm \frac{1}{2}, \theta_{1}\right\rangle \otimes\left|\alpha_{2}= \pm \frac{1}{2}, \theta_{2}\right\rangle$. The two-particle to two-particle $S$-matrix is an operator, $\check{S}: V_{1} \otimes V_{2} \rightarrow V_{2} \otimes V_{1}$. By Lorentz invariance $\breve{S}$ depends only on the combination $\theta_{1}-\theta_{2}$. Apart from the rapidity dependence, $\check{S}$ depends on the coupling $\hat{\beta}$. In order to keep this dependence in mind we denote the two-body $S$-matrix by $\check{S}\left(\frac{x_{1}}{x_{2}} ; q\right)$ with $x_{i}=\exp \left(\theta_{i} / \gamma\right)$ and $q=-\exp (-i \pi / \gamma)$.

The $S$-matrix must commute with the action of the non-local charges since they are symmetries of the theory:

$$
[\check{S}, \Delta(H)]=\left[\check{S}, \Delta\left(Q_{ \pm}\right)\right]=\left[\check{S}, \Delta\left(\bar{Q}_{ \pm}\right)\right]=0 .
$$

Let us rewrite (3.38) in a slightly different form. Representing the charges as in (3.35) and multiplying both sides of (3.38) by $q^{\mathscr{T} / 2} \otimes q^{\mathscr{T} / 2}$ or $q^{-\mathscr{T} / 2} \otimes q^{-\mathscr{T} / 2}$ wherever appropriate, we find:

$$
\begin{aligned}
& {\left[\check{S}\left(\frac{x_{1}}{x_{2}} ; q\right), H \otimes 1+1 \otimes H\right]=0,} \\
& \check{S}\left(\frac{x_{1}}{x_{2}} ; q\right)\left(x_{1} E_{ \pm} \otimes q^{\mp H / 2}+q^{ \pm H / 2} \otimes x_{2} E_{ \pm}\right) \\
& \quad=\left(x_{2} E_{ \pm} \otimes q^{\mp H / 2}+q^{ \pm H / 2} \otimes x_{1} E_{ \pm}\right) \check{S}\left(\frac{x_{1}}{x_{2}} ; q\right), \\
& \check{S}\left(\frac{x_{1}}{x_{2}} ; q\right)\left(x_{1}^{-1} E_{ \pm} \otimes q^{ \pm H / 2}+q^{\mp H / 2} \otimes x_{2}^{-1} E_{ \pm}\right) \\
& \quad=\left(x_{2}^{-1} E_{ \pm} \otimes q^{ \pm H / 2}+q^{\mp H / 2} \otimes x_{1}^{-1} E_{ \pm}\right) \check{S}\left(\frac{x_{1}}{x_{2}} ; q\right) .
\end{aligned}
$$


Jimbo has proven that the solution $\check{S}(x ; q)$ to the above equations is unique up to an overall scalar function $v\left(\theta_{12} \equiv \theta_{1}-\theta_{2}\right)$. He showed that the solution automatically satisfies the Yang-Baxter equation, which is required for factorization of the multiparticle $S$-matrix. Constraints on $v\left(\theta_{12}\right)$ can be found by imposing crossing and unitarity. The minimal solution is the known sine-Gordon $S$-matrix [8]. For completeness we have provided the details in Appendix B.

3g. Alternative Descriptions: The Deformed Gross-Neveu Models. In this section we will describe some of the above results from a different point of view with the objective of further clarifying our construction. As we will see the sine-Gordon theory can be formulated as a deformation of the Gross-Neveu model, or alternatively a deformation of a current-current perturbation of the $S U(2)$ WessZumino-Witten (WZW) model [28].

(i) First consider the above reformulation of the sine-Gordon theory when the coupling $\hat{\beta}=1$. This point is known to correspond to a free Dirac fermion theory. This is easily seen from the equations of motion (3.27), or from the interaction term, if we write it as in (3.30). When $\widehat{\beta}=1$, the $\chi_{ \pm}$fields become the identity and we recover the action and equations of motion for the Dirac theory. Note that the soliton fields (3.23) have spin $\frac{1}{2}$ at this point and are the components of the Dirac fermion. It is interesting to consider what happens to the conserved charges in this limit. The currents (3.12) have Lorentz spin \pm 2 when $\hat{\beta}=1$. They are easily seen to correspond to the energy momentum densities. Namely, $J_{ \pm}$corresponds to the $T_{z z}$ component of the energy-momentum tensor for the topological charge sector \pm 1 . The fields $H_{ \pm}$correspond to the trace of the energy-momentum tensor. The deformation parameter $q$ becomes one in this limit and the algebra (3.19) is abelian; it is just the translation algebra.

(ii) Now consider the theory at the coupling $\widehat{\beta}=\sqrt{2}$, which corresponds to the point where the perturbation is just becoming marginal. The currents (3.12) have dimension one and are nothing other than the vertex operator representation of the level one Kac-Moody currents [29,30]. Of course the usual infinitedimensional Kac-Moody symmetry in the conformal theory is broken in the perturbed theory. What remains is a global $S U(2)$ invariance. In this limit the fields $H_{ \pm}$and $\bar{H}_{ \pm}$become $\bar{J}_{ \pm}$and $J_{ \pm}$respectively. Therefore there are only two conserved currents that results from the above construction (in addition to the topological current) satisfying $\partial_{\bar{z}} J_{ \pm}+\partial_{z} \bar{J}_{ \pm}=0$. The deformation parameter is $q=-1$ when $\hat{\beta}=\sqrt{2}$. In the limit $q=-\lim _{\varepsilon \rightarrow 0} e^{\varepsilon}$ the algebra (3.20) of the non-local charges becomes the global $S U(2)$ algebra.

The limit $\hat{\beta} \rightarrow \sqrt{2}$ in Eqs. (3.39) that characterize the $S$-matrix yields more dynamical information than simply the $s l(2)$-invariance of the $S$-matrix. Define $\varepsilon=1 / \gamma$, and consider the limit $\varepsilon \rightarrow 0$. Keeping terms to order $\varepsilon$, one finds that the equations (3.39) express the Yangian invariance of $\check{S}$ [2]. The reader is referred to Appendix $\mathrm{C}$ for clarification of this point. In particular the Yangian symmetry implies the $S$-matrix at this particular coupling is a rational solution of the Yang-Baxter equation.

When $\hat{\beta}=\sqrt{2}$ the sine-Gordon theory can be described as a current-current perturbation of the level one $S U(2) \mathrm{WZW}$ model. This fact is the origin of the Yangian symmetry of the $S$-matrix at this point, as shown in Appendix C. After 
bosonizing the currents through a vertex operator representation the currentcurrent perturbation becomes the $\cos (\sqrt{2} \Phi)$ perturbation. The $S U(2)$ WZW model at level one is known to contain a single primary chiral field transforming in the fundamental representation [31]. Its scaling dimension is $\frac{1}{4}$. The chiral primary fields correspond precisely to the soliton fields we proposed in (3.23) at $\hat{\beta}=\sqrt{2}$.

A deformation of the current-current perturbation is provided by coupling the different components, $J^{+}, J^{-}$and $J^{0}$ of the Kac-Moody current in an anisotropic way,

$$
S_{D J J}=S_{\mathrm{WZW}}+\frac{\lambda}{2 \pi} \int d^{2} z\left(J^{+} \bar{J}^{-}+J^{-} \bar{J}^{+}+g J^{0} \bar{J}^{0}\right) .
$$

For $g=1$, the action (3.40) describes the current-current perturbation of the $S U(2)$ WZW model, which is of course $S U(2)$ invariant. After bosonization of the level one $S U(2)$ currents, the $J^{0} \bar{J}^{0}$ term which breaks the isotropy amounts to a rescaling of the action of the bosonic fields. This rescaling breaks the $S U(2)$ invariance and re-introduces the coupling constant $\widehat{\beta}$ into the sine-Gordon action.

(iii) Finally let us make contact between the sine-Gordon model and a deformation of the $S U(2)$ Gross-Neveu model. It is well known that at $\hat{\beta}=\sqrt{2}$ the sine-Gordon theory is equivalent to the chiral $S U(2)$ Gross-Neveu model $[30,32]$. Let us deform the $S U(2)$ Gross-Neveu model such that the $S U(2)$ symmetry is broken. The action we choose is,

$$
S_{D G N}=\int \frac{d^{2} z}{4 \pi}\left[\bar{\Psi}_{1} \partial \Psi_{1}+\sigma \bar{\Psi}_{2} \partial \Psi_{2}+\hat{g}\left(\sum_{a=1,2} \bar{\Psi}_{a} \Psi_{a}\right)^{2}-\hat{g}\left(\sum_{a=1,2} \bar{\Psi}_{a} \gamma_{5} \Psi_{a}\right)^{2}\right],
$$

where $\Psi_{a}$ are Dirac fermions. The parameter $\sigma$ in (3.41) measures the deformation away from the $S U(2)$ symmetric point which corresponds to $\sigma=1$. Bosonizing according to the standard rules, $\bar{\Psi}_{a} \Psi_{a}=\cos \left(\varphi_{a}\right)$ and $\bar{\Psi}_{a} \gamma_{5} \Psi_{a}=i \sin \left(\varphi_{a}\right)$, we find,

$$
S_{D G N}=\frac{1}{2 \pi} \int d^{2} z\left[\partial_{z} \varphi_{1} \partial_{\bar{z}} \varphi_{1}+\sigma \partial_{z} \varphi_{2} \partial_{\bar{z}} \varphi_{2}+2 \hat{g} \cos \left(\varphi_{1}-\varphi_{2}\right)\right]
$$

Only the difference of the fields $\varphi_{1}$ and $\varphi_{2}$ interact. We define two new fields $\Phi$ and $\hat{\Phi}$ by the linear combinations,

$$
\Phi=\sqrt{\frac{\sigma}{1+\sigma}}\left(\varphi_{1}-\varphi_{2}\right) ; \quad \hat{\Phi}=\sqrt{\frac{1}{1+\sigma}}\left(\varphi_{1}+\sigma \varphi_{2}\right) .
$$

The kinetic term in (3.42) is the sum of the kinetic terms for the fields $\Phi$ and $\hat{\Phi}$. Therefore, the field $\hat{\boldsymbol{\Phi}}$ decouples, and we are left with a sine-Gordon action with coupling constant $\hat{\beta}=\sqrt{\frac{1+\sigma}{\sigma}}$.

What happens to the Gross-Neveu fermions through this deformation? At the $S U(2)$ invariant point, the standard bosonization formula expresses the fermions as exponentials of the bosonic fields. Namely the components of the Dirac fermions $\Psi_{1}$ are $\psi_{1}=\exp \left(i \phi_{1}\right)$ and $\psi_{1}^{*}=\exp \left(-i \phi_{1}\right)$, where $\phi_{1}$ is the chiral component of $\varphi_{1}$ (and similarly for $\Psi_{2}$ ). Once the deformation parameter $\sigma$ is turned on, expressing everything in terms of the sine-Gordon fields $\Phi$ (and setting the 
decoupled field $\hat{\Phi}$ to zero) we obtain $\psi_{1}=\exp \left(\frac{i}{\hat{\beta}} \phi\right), \psi_{2}=\exp \left(i\left(\hat{\beta}-\frac{1}{\hat{\beta}}\right) \phi\right)$ and similarly for the complex conjugate fields. In other words, we have the following correspondence between the deformation of the Gross-Neveu fermions and the soliton fields (3.23), (3.28),

$$
\begin{array}{ll}
\psi_{1}(x, t)=\Psi_{+}(x, t) ; & \psi_{2}(x, t)=\chi_{-}(x, t) \\
\psi_{1}^{*}(x, t)=\Psi_{-}(x, t) ; & \psi_{2}^{*}(x, t)=\chi_{+}(x, t) .
\end{array}
$$

Hence we see that introducing the parameter $\sigma$ in the $D G N$-action (3.41) breaks the $S U(2)$ symmetry to $\widehat{s l_{q}(2)}$ with $q=\exp (-i 2 \pi \sigma /(1+\sigma))$.

3h. The Restricted Sine-Gordon Theory Revisited. In the works [33-37] it was shown how at special values of the coupling $\hat{\beta}$ one could use the quantum group symmetry of the sine-Gordon $S$-matrix to restrict the theory to obtain perturbations of the $c<1$ minimial conformal series $[38,39]$. It is of value to re-examine the restricted sine-Gordon (RSG) theory from the vantage of the non-local charges developed above.

As emphasized by Reshetikhin and Smirnov [36], the energy momentum tensor is only quantum group invariant if it has a background charge contribution. Now that the charges generating the quantum group symmetry are available, one can see this fact in an explicit way. Let $T(x, t)$ be the $T_{z z}$ component of the energy-momentum tensor. Quantum group invariance of $T(x, t)$ means,

$$
\left[Q_{+}, T(x, t)\right]=0 .
$$

Consider the above equation in the massless limit, when $Q_{+}=\int \frac{d z}{2 \pi i} J_{+}$, with $J_{+}$ given in (3.12a). Equation (3.45) is equivalent to the condition that $Q_{+}$be a dimension zero operator. This is accomplished if $T(x, t)$ has an additional background charge,

$$
T(z, \bar{z})=-\frac{1}{2} \partial_{z} \phi \partial_{z} \phi+i \sqrt{2} \alpha_{0} \partial_{z}^{2} \phi .
$$

That $Q_{+}$has dimension zero is a relation between the background charge $\alpha_{0}$ and the coupling constant $\hat{\beta}$,

$$
1=\frac{2}{\hat{\beta}^{2}}-\frac{2 \sqrt{2}}{\hat{\beta}} \alpha_{0}
$$

When $\hat{\beta}^{2} / 2=p /(p+1), \alpha_{0}$ is such that $c=1-\frac{6}{p(p+1)}$.

In the Feigin-Fuchs description of the minimal models [40,41], Eq. (3.45) defines $Q_{+}$to be a screening operator. Similarly for $\bar{Q}_{-}$. These two screening operators generate the finite dimensional quantum group $s l_{q}(2)$. The other non-local charges $Q_{-}$and $\bar{Q}_{+}$become identified with integrals of the operators $\Phi_{3 ; 1}$ and $\bar{\Phi}_{3 ; 1}$. (See [38] for a definition of these fields.) The spin of these charges is twice the value of when the background charge is zero,

$$
\operatorname{spin}\left(Q_{-}\right)=-\operatorname{spin}\left(\bar{Q}_{+}\right)=\frac{2}{\gamma}=\frac{2}{p} .
$$


In particular this new value of the spin explains why it is necessary to first transform the soliton $S$-matrix from the principal to the homogeneous gradation (see Appendix A) before restricting it. In other words, introducing the background charge in the stress-tensor (3.46) changes the gradation of $\widehat{s l_{q}(2)}$ from the principal to the homogeneous one. The action of the residual conserved charges $Q_{-}$and $\bar{Q}_{+}$on the kink states of the RSG theory were described in [35].

The soliton fields (3.23) are still expected to create particles in the soliton sector of the restricted theory. Let $\Psi_{\alpha= \pm 1 / 2}(x)$ denote the soliton fields $\Psi_{ \pm}(x)$. A multisoliton state is described by

$$
\Psi_{\alpha_{1}}\left(x_{1}\right) \Psi_{\alpha_{2}}\left(x_{2}\right) \cdots \Psi_{\alpha_{N}}\left(x_{N}\right)|0\rangle .
$$

Due to the $s l_{q}(2)$ symmetry, this Hilbert space may be decomposed into irreducible representations

$$
V \otimes \cdots \otimes V=\sum_{j} V^{(j)}
$$

where $V^{(j)}$ is the $2 j+1$ dimensional $\operatorname{spin}(j)$ representation of $s l_{\dot{q}}(2)$, by using the $q$-analog of the Clebsch-Gordon coefficients. We have the following definition of the kink fields $K_{j_{1} j_{2}}(x)$ for $j_{2}=j_{1} \pm 1 / 2$ :

$$
\begin{aligned}
& K_{j_{0} j_{1}}\left(x_{1}\right) K_{j_{1} j_{2}}\left(x_{2}\right) \cdots \\
& \quad=\sum_{\alpha_{1}, \alpha_{2}, m_{1}}\left(\left[\begin{array}{ccc}
j_{0} & \frac{1}{2} & j_{1} \\
\cdot & \alpha_{1} & m_{1}
\end{array}\right]_{q}\left[\begin{array}{ccc}
j_{1} & \frac{1}{2} & j_{2} \\
m_{1} & \alpha_{2} & \cdot
\end{array}\right]_{q} \ldots\right)\left(\Psi_{\alpha_{1}}\left(x_{1}\right) \Psi_{\alpha_{2}}\left(x_{2}\right) \cdots\right),
\end{aligned}
$$

where the brackets are the $q$-Clebsch-Gordon coefficients. The restriction relies on special properties of the representation theory of $s l_{q}(2)$ when $q$ is a root of unity, and amounts to a restriction on the allowed spins of irreducible representations [42-44]. This yields kinks $K_{j_{1} j_{2}}(x)$, where $j_{1}, j_{2} \in\left\{0,1 / 2, \ldots, \frac{p}{2}-1\right\}$.

It is natural to ask where these kink fields are in the minimal models. Including the background charge, the dimensions of the fields $\Psi_{-}, \Psi_{+}$become $\frac{p+3}{4 p}$ and $\frac{p-1}{4 p}$ respectively. The dimension of the field $\Psi_{-}$corresponds to that of the $\Phi_{2,1}$ primary field, whereas the field $\Psi_{+}$is not in the spectrum of primaries.

The minimal unitary models are equivalent to the coset theories $\frac{S U(2)_{1} \otimes S U(2)_{L}}{S U(2)_{1+L}}$ for $p=L+2$, where $S U(2)_{L}$ denotes the $S U(2)$ WZW model at level $L$ [45]. Denote the primary fields of the $S U(2)_{L}$ theory by $(L ; j)$, where $j=0,1 / 2, \ldots, L / 2$. In coset notation the $\Phi_{2,1}$ field is

$$
\Phi_{2,1}(z)=\left[\frac{(1 ; 1 / 2) \otimes(L ; 1 / 2)}{(1+L ; \cdot)}\right](z),
$$

where dot refers to the scalar representation. Thus we see that the $\Phi_{2,1}$ field is associated with the fundamental representation, as expected. The kink fields $K_{j_{1} j_{2}}(x)$ correspond to the intertwiners in the decomposition of $\Phi_{2,1}$ into chiral vertex operators. More specifically, they are associated with the decomposition of the 
factor $(L ; 1 / 2)$. The kink fields thus satisfy the same non-abelian braiding relations as these chiral vertex operators [46-49].

The ultra-violet limit of the RSG theory can be considered as a Liouville theory. We remark that the Liouville theory is also characterized by non-local charges. The Liouville theory has the action

$$
S_{\text {Liouv. }}=\frac{1}{4 \pi} \int d^{2} z \partial_{z} \Phi \partial_{\bar{z}} \Phi+\frac{\lambda}{2 \pi} \int d^{2} z \exp (-i \hat{\beta} \Phi) .
$$

Following the analysis of Sect. 3b, one discovers only two non-local charges $Q_{+}$ and $\bar{Q}_{-}$again defined by (3.12) and (3.13). As described above, requiring these charges to commute with the energy momentum tensor endows it with a background charge, and the charges $Q_{+}$and $\bar{Q}_{-}$have spin zero. Together with the topological charge they satisfy the finite dimensional algebra $s l_{q}(2)$, where $q$ is still given by $q=\exp \left(-2 \pi i / \hat{\beta}^{2}\right)$. These charges may be useful in describing the quantum group symmetry of the Liouville theory and its associated minimal conformal models that has been studied in [50-53].

3i. Additional Remarks. The study of the sine-Gordon theory as a quantum field theory has a long history. It is of interest to compare the above results with the existing literature. Interest in the quantum theory began with Coleman's work, where it was shown that the sine-Gordon theory is equivalent to the massive Thirring model [9]. Coleman further conjectured that the Thirring fermions are the sine-Gordon solitons. Subsequently, Mandelstam constructed quantum operators in the sine-Gordon theory that correspond to the Thirring fermions [10]. On the other hand, Zamolodchikov and Zamolodchikov determined the exact $S$-matrix using a minimal number of assumptions, such as $U(1)$ symmetry, crossing symmetry, the Yang-Baxter equation, and the requirement that it agree with the perturbative expansion [8]. Korepin was able to derive the known $S$-matrix starting from the Thirring theory, but the Thirring fermions had to be treated as pseudo-particles [54]. The true solitons were obtained by filling the Dirac-sea of pseudo-particles in a well-prescribed way.

Within our framework, the fundamental quantum fields that create the solitons have fractional spin $\pm \frac{1}{2 \widehat{\beta}^{2}}$; they can only be identified with the Thirring fermions when $\hat{\beta}=1$, i.e. when the theory is free. Taking into account the expressions for the (anti)-chiral bosonic fields, the soliton fields we consider are of Mandelstamtype. One distinction between our fundamental soliton operators and Mandelstam's fermionic operators is that ours are chiral in the massless limit. Indeed the fermions in the massless Thirring model satisfy $\partial_{\bar{z}} \Psi=\bar{J} \Psi, \partial_{z} \bar{\Psi}=J \bar{\Psi}$, where $J, \bar{J}$ are the $U(1)$ currents. In our notation, the Mandelstam operators are:

$$
\begin{aligned}
& \Psi_{ \pm}^{\text {Mand. }}(x, t)=\exp \left( \pm \frac{i}{2}\left(\frac{1}{\hat{\beta}}+\hat{\beta}\right) \phi(x, t) \mp \frac{i}{2}\left(\frac{1}{\hat{\beta}}-\hat{\beta}\right) \bar{\phi}(x, t)\right), \\
& \bar{\Psi}_{ \pm}^{\text {Mand. }}(x, t)=\exp \left(\mp \frac{i}{2}\left(\frac{1}{\hat{\beta}}+\hat{\beta}\right) \bar{\phi}(x, t) \mp \frac{i}{2}\left(\frac{1}{\hat{\beta}}-\hat{\beta}\right) \phi(x, t)\right) .
\end{aligned}
$$

Note that these operators also have topological charge \pm 1 , and are therefore in the family of fields generated by our fundamental soliton fields. The Mandelstam operators are primarily characterized by their Lorentz spin $\pm 1 / 2$. Thus the 
Mandelstam fermions are not the unique operators that create the solitons. This non-uniqueness can be attributed to the fact that the Lorentz spin of an asymptotic state is not a measurable quantity in $1+1$ dimensions.

\section{Introducing the Center: The Central Sine-Gordon Model}

As we have seen, the algebra of non-local charges in the sine-Gordon theory is $\widehat{s} \widehat{t_{q}(2)}$ with zero center. The fact that the center is zero is what allowed us to find the finite dimensional representations (3.35). These finite dimensional representations were sufficient to deduce the $S$-matrix. One of the lessons learned in the conformal field theories [38] is that it is important for solving the model that the symmetry algebra possess infinite dimensional representations. In the case of the Kac-Moody algebra, it is necessary for the center to be non-zero in order to have only such infinite-dimensional representations. This leads us to believe that one must introduce the center into the algebra $\widehat{s l_{q}(2)}$ in order, for example, to constrain the correlation functions.

The vanishing of the center is traced to the fact there is only one topological charge, which leads to $H_{1}=-H_{0}$ as in (3.22). This suggests that one should introduce new fields into the theory with additional topological charges. It is well-known that the sine-Gordon theory is a Toda theory over the Kac-Moody algebra $\mathrm{sl}(2)$ without center. Namely, introduce a field $\vec{\Omega}$ valued in the Cartan subalgebra of sl(2), $\vec{\Omega}(x, t)=\sum_{i} H_{i} \Phi_{i}(x, t)$, where $H_{i}$ form a basis of the Cartan subalgebra. The duality relation between the simple roots $\alpha_{j}$ of $\widehat{s(2)}$ and the Cartan generators $H_{i}$ is defined through the Cartan matrix: $a_{i j}=H_{i} \cdot \alpha_{j}$. Consider the Toda action,

$$
S_{\text {Toda }}=\frac{1}{4 \pi} \int d^{2} z \frac{1}{2} \partial_{z} \vec{\Omega} \cdot \partial_{\bar{z}} \vec{\Omega}+\frac{\lambda}{2 \pi} \int d^{2} z \sum_{j} \exp \left(\frac{i \hat{\beta}}{\sqrt{2}} \alpha_{j} \cdot \vec{\Omega}\right) .
$$

Setting $\vec{\Omega}=H_{1} \Phi_{1}+H_{0} \Phi_{0}$, where $H_{0}=-H_{1}$, we find that the action (4.1) is the sine-Gordon action for the field $\Phi_{1}-\Phi_{0}$. If we do not impose the zero center condition, but take instead $H_{0}=K-H_{1}$, with $K$ the center, then the resulting action is unaffected since $K \cdot \alpha_{j}=0$.

The correct thing to do is to recognize that the full algebra sl(2) contains an additional element, the derivation, which we denote by $d$. The derivation measures the order of the grading. $\left[d, J_{n}^{a}\right]=n J_{n}^{a}$. With the derivation included, the Killing form is non-degenerate on the Cartan subalgebra. In particular the important non-zero inner product is $\langle d, K\rangle=2$. Following Babelon and Bonora [55], we introduce an additional field $\eta$ for the derivation,

$$
\vec{\Omega}(x, t)=H \Phi(x, t)+d \eta(x, t)+K \xi(x, t) .
$$

The affine Toda action (4.1) becomes:

$$
S_{\mathrm{CSG}}=\frac{1}{4 \pi} \int d^{2} z\left(\partial_{z} \Phi \partial_{\bar{z}} \Phi+\frac{1}{2}\left(\partial_{z} \eta \partial_{\bar{z}} \xi+\partial_{\bar{z}} \eta \partial_{z} \xi\right)\right)+\frac{\lambda}{2 \pi} \int d^{2} z\left(e^{i \hat{\beta} \Phi}+e^{-i \hat{\beta} \Phi+i \hat{\beta} \eta}\right) .
$$

Setting $\eta$ to zero, one recovers the sine-Gordon theory. 
Let us now see the implication for the non-local charges. A simple computation, that we leave to the reader, shows that four non-local currents are still conserved. The currents $J_{ \pm}, \bar{J}_{ \pm}$and $H_{-}, \bar{H}_{+}$are defined as before, (3.12). Only the currents $H_{+}$and $\bar{H}_{-}$are changed according to:

$$
\begin{aligned}
& H_{+}(x, t)=-\gamma \lambda \exp \left[i\left(\frac{2}{\hat{\beta}}-\hat{\beta}\right) \phi(x, t)-i \hat{\beta} \bar{\phi}(x, t)+i \hat{\beta} \eta(x, t)\right], \\
& \bar{H}_{-}(x, t)=-\gamma \lambda \exp \left[i\left(\frac{2}{\hat{\beta}}-\hat{\beta}\right) \bar{\phi}(x, t)-i \hat{\beta} \phi(x, t)+i \hat{\beta} \eta(x, t)\right] .
\end{aligned}
$$

The non-local charges now satisfy the following algebra:

$$
\begin{aligned}
Q_{ \pm} \bar{Q}_{ \pm}-q^{2} \bar{Q}_{ \pm} Q_{ \pm} & =0, \\
Q_{+} \bar{Q}_{-}-q^{-2} \bar{Q}_{-} Q_{+} & =a\left(1-q^{2 \mathscr{T}+2 \mathscr{T}} \eta\right), \\
Q_{-} \bar{Q}_{+}-q^{-2} \bar{Q}_{+} Q_{-} & =a\left(1-q^{-2 \mathscr{T}}\right),
\end{aligned}
$$

$\left(a \equiv \frac{\lambda}{2 \pi i} \gamma^{2}\right)$ where $\mathscr{T}_{n}$ is the topological charge of the $\eta$ field,

$$
\mathscr{T}_{n}=-\frac{\hat{\beta}^{3}}{4 \pi} \int d x \partial_{x} \eta
$$

This is the centered $\widehat{s l_{q}(2)}$ algebra with $H_{0}=-\mathscr{T} ; H_{1}=\mathscr{T}+\mathscr{T}_{n}$.

We conclude that the central sine-Gordon models are invariant under the quantum affine Kac-Moody algebra $\widehat{s l_{q}(2)}$, and the center of $\widehat{s_{q}(2)}$ is the topological charge of the additional field $\eta$. This suggests that it could be possible to formulate the central sine-Gordon model as a theory of interacting $\widehat{s_{q}(2)}$ modules.

\section{Generalization to Other Groups}

In this section we will generalize the above results to other groups. Namely, we will construct the non-local charges for the affine Toda theories based on a simply laced Lie algebra $G$. These results will then be applied to perturbations of conformal field theories. We skip some of the details, having provided them for the sl(2) case.

5a. Non-Local Charges for the Affine Toda Theories. Let $G$ be a simply-laced finite dimensional Lie algebra of rank $r$, and $\hat{G}$ its affine extension. In this section, $\hat{G}$ and its $q$-deformation $\hat{G}_{q}$ will always refer to the zero-center algebras. A basis of simple roots for $\hat{G}$ will be denoted by $\vec{\alpha}_{i}, i=0,1, \ldots, r$, where $\vec{\alpha}_{i=1, \ldots, r}$ are the simple roots of $G$ and $\vec{\alpha}_{0}$ is the extended root, i.e. the horizontal projection of $\vec{\alpha}_{0}$ is the negative of the highest root of $G$. For simply-laced algebras, the roots all have the same length $\vec{\alpha}_{i} \cdot \vec{\alpha}_{i}=2$. Let $\vec{\Phi}$ be a vector of fields valued in the Cartan subalgebra of the algebra $\widehat{G}$, i.e. $\vec{\Phi}=\left(\Phi^{1}, \Phi^{2}, \ldots, \Phi^{r}\right)$. The affine Toda theory is defined by the action

$$
S=\frac{1}{4 \pi} \int d^{2} z \partial_{z} \Phi \cdot \partial_{\bar{z}} \Phi+\frac{1}{2 \pi} \int d^{2} z \sum_{\alpha_{j} \text { simple }} \exp \left(-i \frac{\hat{\beta}}{\sqrt{2}} \alpha_{j} \cdot \Phi\right)
$$


where $\hat{\beta}$ is a real coupling in the range $0 \leqq \hat{\beta} \leqq \sqrt{2}$. For convenience of notation we define $\tilde{\beta} \equiv \hat{\beta} / \sqrt{2}$. In the above equation, the sum is over the simple roots of $\hat{G}$. The propagator for the fields are

$$
\left\langle\Phi^{i}(z, \bar{z}) \Phi^{j}(0)\right\rangle=-\delta^{i j} \log (z \bar{z}) .
$$

The sine-Gordon theory is equivalent to the $\widehat{s(2)}$ case. As described for the sine-Gordon theory, we treat the action (5.1) as a perturbation of the free boson theory. The chiral and anti-chiral components $\phi^{i}$ and $\bar{\phi}^{i}$ of the Toda fields are defined as in (3.4).

The action (5.1) is not hermitian unless the algebra is $\operatorname{sl}(2)$. It is also not renormalizable [56]. These problems will be addressed in Sect. 5c. Our aim is to identify non-local conserved charges in this model and to show how they provide a non-perturbative scheme for quantizing the Toda theory (5.1). In other words, our approach consists in defining the theory by implementing the non-local symmetry.

Following the analysis in Sect. 3b, we find the following non-local conserved charges to first order in perturbation:

$$
\partial_{\bar{z}} J_{\alpha_{j}}=\partial_{z} H_{\alpha_{j}} ; \quad \partial_{z} \bar{J}_{-\alpha_{j}}=\partial_{\bar{z}} \bar{H}_{-\alpha_{j}},
$$

with,

$$
\begin{aligned}
J_{\alpha_{j}}(x, t) & =\exp \left(\frac{i}{\tilde{\beta}} \alpha_{j} \cdot \phi(x, t)\right), \\
H_{\alpha_{j}}(x, t) & =-\gamma \lambda \exp \left(-i\left(\tilde{\beta}-\frac{1}{\tilde{\beta}}\right) \alpha_{j} \cdot \phi(x, t)-i \tilde{\beta} \alpha_{j} \cdot \bar{\phi}(x, t)\right), \\
\bar{J}_{-\alpha_{j}}(x, t) & =\exp \left(\frac{i}{\tilde{\beta}} \alpha_{j} \cdot \bar{\phi}(x, t)\right), \\
\bar{H}_{-\alpha_{j}}(x, t) & =-\gamma \lambda \exp \left(-i\left(\tilde{\beta}-\frac{1}{\tilde{\beta}}\right) \alpha_{j} \cdot \bar{\phi}(x, t)-i \tilde{\beta} \alpha_{j} \cdot \phi(x, t)\right) .
\end{aligned}
$$

In the above equation, $\alpha_{j}$ is any simple root of $\hat{G}$. The above currents define $r+1$ conserved charges

$$
\begin{aligned}
Q_{\alpha_{j}} & =\frac{1}{2 \pi i}\left(\int d z J_{\alpha_{j}}+\int d \bar{z} H_{\alpha_{j}}\right), \\
\bar{Q}_{-\alpha_{j}} & =\frac{1}{2 \pi i}\left(\int d \bar{z} \bar{J}_{-\alpha_{j}}+\int d z \bar{H}_{-\alpha_{j}}\right) .
\end{aligned}
$$

As before, the spin of these charges is $\pm 1 / \gamma$, where $\gamma$ is defined in (3.14): $\frac{1}{\gamma}=\frac{1}{\widetilde{\beta}^{2}}-1=$ $\frac{2}{\hat{\beta}^{2}}-1$. We define the topological charges

$$
H_{i} \equiv H_{\alpha_{i}}=\frac{\tilde{\beta}}{2 \pi} \int_{t} d x \alpha_{i} \cdot \partial_{x} \Phi
$$


Due to the fact that there are only $r$ independent fields, the above $r+1$ topological charges are not all linearly independent.

The conserved charges satisfy the following algebra:

$$
\begin{aligned}
{\left[H_{\alpha_{i}}, Q_{\alpha_{j}}\right] } & =a_{i j} Q_{\alpha_{j}}, \\
{\left[H_{\alpha_{i}}, \bar{Q}_{-\alpha_{j}}\right] } & =-a_{i j} \bar{Q}_{-\alpha_{j}}, \\
Q_{\alpha_{i}} \bar{Q}_{-\alpha_{k}}-q^{\alpha_{j k}} \bar{Q}_{-\alpha_{k}} Q_{\alpha_{j}} & =a \delta_{j k}\left(1-q^{\left.2 H_{\alpha_{j}}\right),}\right.
\end{aligned}
$$

$\left(a \equiv \frac{\lambda}{2 \pi i} \gamma^{2}\right)$ where $q$ is as in (3.18), $q=\exp \left(-i \pi / \widetilde{\beta}^{2}\right)$, and $a_{i j}=\alpha_{i} \cdot \alpha_{j}$ is the generalized Cartan matrix of $\hat{G}$. These equations are the defining relations of the $\hat{G}_{q}$ loop algebra. Only the Serre relations are missing. (See Appendix A for definitions.) The relation with the generators $E_{i}, F_{i}, H_{i}$ of $\hat{G}_{q}$ is

$$
Q_{\alpha_{i}}=c E_{i} q^{H_{i} / 2} ; \quad \bar{Q}_{-\alpha_{i}}=c F_{i} q^{H_{i} / 2}
$$

for some constant $c$.

5b. The Soliton Fields and the S-Matrices. Let us first consider the $S U(N)$ case. The soliton fields are characterized by their locality with respect to the perturbation; this ensures they have well-defined equations of motion. These soliton fields are in one-to-one correspondence with the $N-1$ fundamental representations of $S U(N)$. For $S U(N)$, all the fundamental representations are integrable (highest weight) representations of $\hat{G}$ at level one. We therefore have a simple vertex operator representation of these soliton fields. The soliton fields associated to the $n^{\text {th }}$ fundamental representation are:

$$
\begin{aligned}
& \Psi_{\mu_{n}}(x, t)=\exp \left(\frac{i}{\tilde{\beta}} \mu_{n} \cdot \phi(x, t)\right), \\
& \bar{\Psi}_{\mu_{n}}(x, t)=\exp \left(-\frac{i}{\tilde{\beta}} \mu_{n} \cdot \bar{\phi}(x, t)\right),
\end{aligned}
$$

with $\mu_{n}$ any weight of the $n^{\text {th }}$ fundamental representation of $S U(N)$. The topological charge of $\Psi_{\mu_{n}}$ or $\bar{\Psi}_{\mu_{n}}$ is the weight $\mu_{n}$. As for $S U(2)$, all the fields which differ from the soliton fields (5.9) by local operators have the same topological charge. They also create the solitons asymptotically.

We denote by $\rho_{n}$ the $n^{\text {th }}$ fundamental representation, by $V_{n}$ its representation vector space, by $\omega_{n}$ its highest weight (satisfying $\omega_{n} \cdot \alpha_{j}=\delta_{n j}$ ), and by $\left[\omega_{n}\right]$ the set of weights of $\rho_{n}$.

The fields whose topological charges are weights in $\left[\omega_{n}\right]$ are stable under the action of the non-local charges (5.5). More precisely, we have:

$$
\begin{array}{clll}
Q_{\alpha_{j}}\left(\bar{\Psi}_{\mu_{n}}\right)=0 & \text { if } & \left(\mu_{n}+\alpha_{j}\right) \notin\left[\omega_{n}\right], \\
Q_{\alpha_{j}}\left(\bar{\Psi}_{\mu_{n}}\right)=\lambda \hat{\Psi}_{\mu_{n}+\alpha_{j}} & \text { if } & \left(\mu_{n}+\alpha_{j}\right) \in\left[\omega_{n}\right], \\
\bar{Q}_{-\alpha_{j}}\left(\Psi_{\mu_{n}}\right)=0 & \text { if } & \left(\mu_{n}-\alpha_{j}\right) \notin\left[\omega_{n}\right], \\
\bar{Q}_{-\alpha_{j}}\left(\Psi_{\mu_{n}}\right)=\lambda \hat{\Psi}_{\mu_{n}-\alpha_{j}} & \text { if } & \mu_{n}-\alpha_{j} \in\left[\omega_{n}\right],
\end{array}
$$

where $\hat{\Psi}_{\mu_{n}}$ (or $\hat{\bar{\Psi}}_{\mu_{n}}$ ) are fields which differ from $\Psi_{\mu_{n}}$ (or $\bar{\Psi}_{\mu_{n}}$ ) by local operators. Note that (5.10) is compatible with the weight structure of $S U(N)$. 
Thus we conclude there are $N-1$ families of solitons, where each family transforms under a fundamental representation of $\widehat{S U_{q}(N)}$. We will refer to these representations as $\hat{\rho}_{n}$, and to the vector spaces of these representations as $W_{n}$. The solitons in a given family are all degenerate in mass. We define $M_{n}$ to be the mass of the solitons in the representation $\hat{\rho}_{n}$. These masses will be specified below.

The action of the non-local charges on the asymptotic solitons can be deduced from (5.10), from the fact that they must form a representation of the algebra $\widehat{S U_{q}(N)}$, and from the Lorentz spin of the charges. As can be seen from (5.10), the vector space $W_{n}$ of the representation $\hat{\rho}_{n}$ of $\widehat{S U_{q}(N)}$ is isomorphic to the vector space $V_{n}$ of the representation $\rho_{n}$ of $S U(N): W_{n} \equiv V_{n}$. The action of the non-local charges on asymptotic states in $\hat{\rho}_{n}$ are given by (5.8), where the generators $E_{i}, F_{i}, H_{i}$ of $\widehat{S U_{q}(N)}$ are in the principal gradation of the representation $\hat{\rho}_{n}$. More specifically,

$$
Q_{\alpha_{i}}=c e^{\theta / \gamma} \hat{e}_{i} q^{H_{i} / 2} ; \quad \bar{Q}_{-\alpha_{i}}=c e^{-\theta / \gamma} \hat{f}_{i} q^{H_{i} / 2},
$$

where $\hat{e}_{i}, \hat{f}_{i}, H_{i}$ form a spectral parameter independent representation of the quantum algebra $\widehat{S U_{q}(N)}$ on the vector space $V_{n} \equiv W_{n}$.

Let $\breve{S}_{m n}$ denote the $S$-matrix for the scattering of two incoming (and outgoing) particles in the representations $\hat{\rho}_{m}, \hat{\rho}_{n}$. It is an operator $\breve{S}_{m n}: V_{m} \otimes V_{n} \rightarrow V_{n} \otimes V_{m}$. This $S$-matrix is required to commute with the action of the non-local charges. This is equivalent to the equations

$$
\left[\check{S}_{m n},\left(\hat{\rho}_{m} \otimes \hat{\rho}_{n}\right) \Delta\left(E_{i}\right)\right]=\left[\check{S}_{m n},\left(\hat{\rho}_{m} \otimes \hat{\rho}_{n}\right) \Delta\left(F_{i}\right)\right]=\left[\check{S}_{m n},\left(\hat{\rho}_{m} \otimes \hat{\rho}_{n}\right) \Delta\left(H_{i}\right)\right]=0,
$$

where $\Delta$ is the standard comultiplication for $\widehat{S U_{q}(N)}$.

It has been shown that the solutions $\tilde{S}_{m n}$ to (5.12) are unique up to an overall scalar function. Standard techniques have been developed for finding the solutions. We refer the reader to a recent review of Jimbo [57]. We take the following ansatz for $\check{S}_{m n}$ :

$$
\check{S}_{m n}(\theta)=X_{m n}(\theta) v_{m n}(\theta, q) \check{R}_{m n}(\theta, q),
$$

(see the analysis in Appendix B for the $S U(2)$ case). Above, $\check{R}_{m n}$ is a crossing symmetric solution to (5.12). The scalar function $v_{m n}$ is defined to be the minimal solution to the constraints of crossing and unitarity for the product $v_{m n} \tilde{R}_{m n}$. The unitarity constraint reads

$$
\left(v_{m n}(\theta, q) v_{n m}(-\theta, q)\right)^{-1}=\check{R}_{m n}(\theta, q) \check{R}_{n m}(-\theta, q) .
$$

The crossing symmetry condition on $v_{m n}$ is

$$
v_{m n}(\theta)=v_{\bar{n} m}(i \pi-\theta)
$$

where $\bar{n}$ is the charge-conjugate representation to $n$.

The additional factor $X_{m n}(\theta)$ is a CDD ambiguity factor that is by itself a solution to the unitarity and crossing conditions. The mass $M_{n}$ and the CDD factors will be fixed below (see Sect. 5c) by examining the $\widehat{\beta} \rightarrow \sqrt{2}$ limit. They will turn out to be the same as for the $S U(N)$ Gross-Neveu models [58, 59]. To clarify, we first give the result; the masses are determined to be:

$$
M_{n}=M \frac{\sin (n \pi / N)}{\sin (\pi / N)} ; \quad n=1, \ldots, N-1,
$$


and the CDD factors $X_{m n}$ are:

$$
X_{m n}(\theta)=f(m+n) f(m+n-2)^{2} f(m+n-4)^{2} \cdots f(|m-n|),
$$

where

$$
f(x)=\frac{\sinh \left(\frac{\theta}{2}+\frac{i \pi x}{2 N}\right)}{\sinh \left(\frac{\theta}{2}-\frac{i \pi x}{2 N}\right)} .
$$

The $S$-matrix $(5.13)$ was conjectured in $[11,12]$. That it satisfies all the necessary requirements has been checked in [12].

Let us now turn to the other simply laced algebras $G$. We use the same convention for denoting the representations of $G$ and $\hat{G}_{q}$. New subtleties arise due to the fact that not all the soliton fields admit a vertex operator representation as in (5.9). Only the solitons which belong to the fundamental representations of $G$ that are integrable for $\hat{G}$ at level one have such simple soliton fields. Let $\vec{\theta}=\sum_{i=1}^{r} m_{i} \vec{\alpha}_{i}$, where $\vec{\theta}$ is the highest root, and $m_{i}$ is a positive integer. The fundamental representations that are integrable at level one are $\rho_{i}$ for which $m_{i}=1$. Such representations exist for all the algebras except $E_{8}$. For this class of representations, all the computations described above can be similarly done. This implies that for these specific fundamental representations we still have the isomorphism between the spaces $W_{n}$ and $V_{n}: W_{n} \equiv V_{n}$. For the other representations, the situation is more complicated. The spaces $W_{n}$ and $V_{n}$ are no longer isomorphic; $W_{n}$ is the direct sum of a finite number of spaces $V_{k}$. This can be seen in a variety of ways. For the approach we are developing, this fact is best thought of as a property of the representation theory for the algebra $\hat{G}_{q}$. Namely, the representations of $\hat{G}_{q}$ are not isomorphic to that of $G_{q} \otimes C\left(x, x^{-1}\right)$, where $C\left(x, x^{-1}\right)$ is an arbitrary function of the spectral parameter $x$. The difference between the spaces $W_{n}$ and $V_{n}$ finds it origin in the Serre relations for the quantum loop algebras $\hat{G}_{q}$. This can be explicitly seen by examining the fusion procedure [60] for the $\hat{G}_{q} \breve{R}_{\text {matrices. }}$ Alternatively, this fusion procedure may be viewed as nothing other than the requirement of closure of the bootstrap for factorizable scattering. In all cases the $S$-matrix will still be as in (5.13) but now it acts on the spaces $W_{m} \otimes W_{n}$ :

$$
\begin{aligned}
& \breve{S}_{m n}^{G}: W_{m} \otimes W_{n} \rightarrow W_{n} \otimes W_{m}, \\
& \breve{S}_{m n}^{G}(\theta)=X_{m n}^{G}(\theta) v_{m n}^{G}(\theta, q) \breve{R}_{m n}^{G}(\theta, q),
\end{aligned}
$$

where $\breve{R}_{m n}^{G}$ are the $\hat{G}_{q} \breve{R}$-matrices in the representation $W_{m} \otimes W_{n}$.

Let us be more specific by specializing to the $D_{N}=S O(2 N)$ case. The fundamental representations which are integrable at level one are the vector and the spinor representations. We denote by $\left(\rho_{s}, V_{s}\right)$ and $\left(\rho_{\bar{s}}, V_{\bar{s}}\right)$ the spinor representations and their vector spaces, and by $\left(\rho_{n}, V_{n}\right), n=1, \ldots, N-2$, the tensorial representations ordered in the usual way; $\rho_{1}$ is the vector representation. To illustrate how the difference between the spaces $V_{n}$ and $W_{n}$ manifests itself, consider the $\widehat{S O_{q}(2 N)} R$ matrix in the vector representation and its fusion. In the homogeneous gradation 
(for simplicity) this $R$ matrix is [57]:

$$
\begin{aligned}
\breve{R}_{V_{1} \otimes V_{1}}(x)= & \left(q^{2 N-2} x-1\right)\left(q x-q^{-1}\right) P_{2 \omega_{1}}-\left(q^{2 N-2} x-1\right)\left(q^{-1} x-q\right) P_{\omega_{2}} \\
& +\left(x-q^{2 N-2}\right)\left(q^{-1} x-q\right) P_{\omega_{0}},
\end{aligned}
$$

where $P_{\omega}$ are the projectors onto the irreducible representations of $S O_{q}(2 N)$ of highest weight $\omega$.

To apply the fusion procedure [60], we first have to find a value of $x$ for which $\breve{R}_{V_{1} \otimes V_{1}}$ projects onto a proper subspace of $V_{1} \otimes V_{1}$. For $x q^{2 N-2}=1, \breve{R}_{V_{1} \otimes V_{1}}$ projects onto the trivial representation. However for $x q^{2}=1$, we have

$$
\breve{R}_{V_{1} \otimes V_{1}}\left(q^{-2}\right) \propto\left(q^{N-2}-q^{2-N}\right) P_{\omega_{2}}+\left(q^{N}-q^{-N}\right) P_{\omega_{0}} .
$$

This implies there is no way to disentangle the space $V_{2}$ from the space $V_{0}$. Therefore the fusion procedure shows that $W_{2}=V_{2}+V_{0}$ and that

$$
\breve{R}_{V_{1} \otimes W_{2}}(x)=\left.\left(1 \otimes \breve{R}_{V_{1} \otimes V_{1}}(x q)\right)\left(\breve{R}_{V_{1} \otimes V_{1}}\left(x q^{-1}\right) \otimes 1\right)\right|_{V_{1} \otimes W_{2}} .
$$

This method goes through for the other fundamental representations by fusing recursively. The decomposition of the spaces $W_{n}$ on the spaces $V_{n}$ and the masses are:

$$
\begin{array}{ll}
W_{n}=\bigoplus_{k=0}^{[n / 2]} V_{n-2 k} ; & M_{n}=2 M \sin \left(\frac{n \pi}{2 N-2}\right) \text { for } n=1, \ldots, N-2 ; \\
W_{s}=V_{s} ; & M_{s}=M ; \\
W_{\bar{s}}=V_{\bar{s}} ; & M_{\bar{s}}=M .
\end{array}
$$

In all cases the decompositions of the spaces $W_{n}$ coincide with those of the representations of the Yangians. This is because the $S$-matrix (5.19) is Yangian invariant in the limit $\hat{\beta}=\sqrt{2}$. This will be explained in the next section, but could have been foreseen from the idea the spaces $W_{n}$ are expected to be stable when $\hat{\beta}$ is varying. The depositions of the spaces $W_{n}$ and the masses $M_{n}$ are all known and can be found in $[61,59]$.

Though we have fixed some of the properties of the $S$-matrix by appealing to known results on the Gross-Neveu-like models, this is not a necessary feature of our construction; these results could have been found by closing the bootstrap directly.

5c. The Gross-Neveu Limit. As shown in [56], the theory (5.1) is not renormalizable. Let us demonstrate this fact by a different analysis than the one used in [56] that is more suited to our purpose. Consider the action (5.1) when $\hat{\beta}=\sqrt{2}$. At this point the perturbing operators are marginal. More generally, consider a conformal field theory perturbed by some marginal operators $\mathcal{O}^{i}(z, \bar{z})$,

$$
S=S_{\mathrm{CFT}}+\frac{1}{2 \pi} \int d^{2} z \sum_{i} \lambda_{i} \mathcal{O}^{i}(z, \bar{z}) \text {. }
$$

Introducing an cutoff $l$, Zamolodchikov has shown [62] that the beta-functions to second order are

$$
\frac{d \lambda_{i}}{d \log (l)}=-C_{i}^{j k} \lambda_{j} \lambda_{k}+\cdots
$$


where $C_{i}^{j k}$ are the structure constants of the operator algebra:

$$
\mathcal{O}^{i}(z, \bar{z}) \mathcal{O}^{j}(0) \sim C_{k}^{i j} \frac{\mathcal{O}^{k}(0)}{|z|^{2}}+\cdots
$$

Returning to the action (5.1), the perturbing operator is $\sum_{\alpha_{\text {simple }}} J_{\alpha} \bar{J}_{-\alpha}$, where $J_{\alpha}=\exp (i \alpha \cdot \phi)$ is the bosonized expression for the level one Kac-Moody current $[29,30]$. The main point is that the operators $J_{\alpha}$ for $\alpha$ a simple root obviously do not form a closed algebra by themselves; a closed set of operators is given by $J_{ \pm \alpha}$ for any positive root $\alpha$ (along with $H_{\alpha}$ ). From (5.26) and (5.24) we thus infer that operators corresponding to the non-simple roots will be generated under renormalization. We are thus led to the current-current perturbation of the level one WZW model:

$$
S=S_{\mathrm{wZw}}+\frac{\lambda}{2 \pi} \int d^{2} z \sum_{a} J^{a} \bar{J}^{a},
$$

where $J^{a}, \bar{J}^{a}$ are the Kac-Moody currents. This action is now renormalizable. From the bosonized form of the Kac-Moody currents, one sees that the above action (5.27) is of Gross-Neveu type:

$$
S=\frac{1}{4 \pi} \int d^{2} z \partial_{z} \Phi \cdot \partial_{\bar{z}} \Phi+\frac{\lambda}{\pi} \int d^{2} z \sum_{a>0} \cos (\tilde{\beta} \alpha \cdot \Phi) ; \quad \text { with } \tilde{\beta}=1,
$$

where the sum extends over all positive roots. For example, the bosonization of the $S U(N)$ Gross-Neveu models yields the above action [30,32]. (See Sect. 3g for the case of $S U(2))$.

The $S$-matrices for the $G$-invariant Gross-Neveu-like models (5.28) are known explicitly for the classical Lie algebras $[58,59]$. The structure of these $S$-matrices is expected to be the same for all Lie algebras. We now argue that the $\hat{\beta} \rightarrow \sqrt{2}$ limit of the $S$-matrices in (5.13) are precisely these $S$-matrices. Note that the nonlocal currents in (5.4) are the bosonized expressions for the Kac-Moody currents in this limit. Furthermore, in this limit $q \rightarrow-1$, and the symmetry algebra is isomorphic to the Lie algebra $G$. Thus the $S$-matrix must be $G$-invariant. The fact that the theory can be formulated as a current-current perturbation of the WZW model further implies that the theory has Yangian symmetry (see Appendix C). This symmetry can be seen explicitly by taking the limit $\widehat{\beta} \rightarrow \sqrt{2}$ carefully in Eqs. (5.12), as is shown in Appendix $C$ for the $S U(2)$ case.

For other values of the coupling $\hat{\beta}$ near $\sqrt{2}$, the arguments based on the beta-functions (5.25) are not significantly modified, and one therefore expects the action to still be unrenormalizable. However, at this stage of development we are unable to conclude that the $S$-matrices that are solutions to (5.12) correspond to the theory with the action (5.28), except at $\hat{\beta}=\sqrt{2}$. The reason is that the nonlocal charges (5.5) are not symmetries of (5.28), but are symmetries of (5.1).

To summarize this section, we have found that for $\hat{\beta}<\sqrt{2}$, the $S$-matrices (5.13) can only be formally associated with the action (5.1) due to the nonrenormalizability and non-hermiticity of the action. At $\hat{\beta}=\sqrt{2}$, the $S$-matrix (5.13) is the physical $S$-matrix for the renormalizable action (5.28). However, as we will 
see in the next section, the $S$-matrices (5.13) are just what one requires in order to solve certain perturbations of the coset conformal field theories.

5d. Applications to Perturbed Coset Conformal Field Theories. Let $G_{K}$ denote the $G$-invariant WZW model at level $K$. In [11] factorizable $S$-matrices for the full series of coset conformal field theories $G_{K} \otimes G_{L} / G_{K+L}$ were conjectured. The main difficulty in proving these conjectures is establishing the form of the "unrestricted" $S$-matrix (for $S U(2)$ this is just the sine-Gordon soliton $S$-matrix). In this section the non-local charge formalism will be used to determine these $S$-matrices.

We consider the coset conformal field theories $G_{1} \otimes G_{L} / G_{1+L}$ with central charge [45]

$$
c=c\left(G_{1}\right)+c\left(G_{L}\right)-c\left(G_{1+L}\right) .
$$

It is well-known that the coset CFT has a generalized Feigin-Fuchs construction consisting of $r$ bosonic fields $\vec{\Phi}$ valued in the Cartan sub-algebra of $G[63,64]$. The energy momentum tensor takes the form

$$
T(z)=-\frac{1}{2} \partial_{z} \Phi \cdot \partial_{z} \Phi+i \alpha_{0} \vec{\rho} \cdot \partial_{z}^{2} \vec{\Phi}
$$

where $\vec{\rho}$ is the Weyl vector of $G$, and $\alpha_{0}$ is a background charge

$$
\alpha_{0}=\frac{1}{\sqrt{\left(L+h^{*}+1\right)\left(L+h^{*}\right)}} .
$$

( $h^{*}$ equals the dual coxeter number of $G$ ). The Feigin-Fuchs construction can be formulated as the quantization of the simple Toda theory over the Lie algebra $G$ [63]. The simple Toda action is as in (5.1), but without the extended affine root in the sum. To formulate the Feigin-Fuchs construction in this way, one chooses the coupling $\frac{\hat{\beta}}{\sqrt{2}}$ to be a screening charge: $\frac{\hat{\beta}}{\sqrt{2}}=-\alpha_{-}$, i.e.

$$
\frac{\hat{\beta}}{\sqrt{2}}=\sqrt{\frac{L+h^{*}}{L+h^{*}+1}} .
$$

As recognized in $[65,66]$, if one treats the additional extended root term in the affine Toda theory as a perturbation of the CFT of the simple Toda theory, then it formally amounts to a perturbation by the coset field

$$
\Phi_{\text {pert }}(z, \bar{z})=\left[\frac{(1 ; \cdot) \otimes(L ; \cdot)}{(1+L ; \operatorname{Adj})}\right](z, \bar{z}),
$$

where $(L ; \Lambda)$ is an integrable representation of highest weight $\Lambda$ for $G_{K}$, and $\cdot$, Adj refer to the scalar and adjoint representations respectively.

It is important to bear in mind that the Feigin-Fuchs construction entails a restriction of the operator algebra, i.e. the null states must be projected out $[40,41]$. Upon this restriction the unitary, rational CFT of the coset is obtained. This implies that the action in (5.1) is not to be taken literally for the applications we have in mind. It is precisely this kind of restriction of the operator algebra that is needed to render invalid the argument of Sect. $5 \mathrm{~b}$ that led us to conclude that the action is not renormalizable. Therefore we conclude that when $L$ is finite, no 
additional terms are generated in the action (5.1) under renormalization, due to the above restriction of the operator algebra. In particular this implies that the non-local charges (5.5) can be used to solve the model. Note that when $L \rightarrow \infty$, there is no restriction of the operator algebra and we are back to the Gross-Neveu action (5.28).

As for the $s l(2)$ case $[33,35,36]$, the $S$-matrices (5.19) must be restricted to the RSOS form in order to describe the perturbed coset theories. (See also [67] for the case of restriction of the twisted $A_{2}^{(2)}$ theory.) This restriction is a massive analog of the Feigin-Fuchs construction. The restriction procedure relies on special properties of quantum group representations for $q$, which now is $\exp \left(-i \pi /\left(L+h^{*}\right)\right)$ from Eqs. (3.18) and (5.32), equal to a root of unity. Standard techniques have been developed for performing this computation $[68,69]$. More specifically, the $S$-matrix for the $L^{\text {th }}$ theory in the coset series was conjectured to be [11]

$$
\breve{S}_{m n}^{[L]}(\theta)=X_{m n}(\theta) v_{m n}(\theta, q) \breve{R}_{m n}^{[L]}(\theta, q),
$$

where $\breve{R}_{m n}^{[L]}$ is the crossing symmetric RSOS restriction of $\breve{R}_{m n}$. (We have dropped the superscript $G$ in (5.19).)

The spectrum consists of $r$ families of kinks, which we denote as $\left(K_{n}\right), n=1, \ldots, r$, and all kinks in the $n^{\text {th }}$ family have the same mass $M_{n}$. For concreteness we now specialize to $S U(N)$. The kinks in $\left(K_{n}\right)$ are labelled by two highest weights of $S U(N)$. A kink of rapidity $\theta$ is $K_{n}^{[a \rightarrow b]}(\theta)$, where $a, b$ denote highest weight representations of the $S U(N)$ WZW model at level $L$. A pair of weights $[a \rightarrow b]$ is said to be $n$-admissible iff the representation $b$ appears in the tensorial product $a \otimes \rho_{n}$. Only $n$-admissible pairs of $[a \rightarrow b]$ appear in the kinks of the family $\left(K_{n}\right)$.

When $L=1$, as in the $S U(2)$ case the RSOS quantum numbers are frozen, and the $S$-matrix reduces to the CDD factors:

$$
\breve{S}_{m n}^{[1]}(\theta)=X_{m n}(\theta) \text {. }
$$

This is the result conjectured in [70]. In this context, the CDD factors are referred to as the minimal solutions to the bootstrap for the spectrum of masses $M_{n}$. They also appear in the study of real coupling affine Toda theory and can be found in $[71]^{4}$.

\section{Conclusions}

To summarize, we have demonstrated that quantum non-local conserved charges characterize many integrable quantum field theories. These non-local conserved currents provide a quantum field theoretic basis for understanding the structure of Quantum Groups.

Though our discussion in Sect. 5 has focused on the integrable quantum field theories related to perturbations of the cosets where one of the levels is one, the methods extend to the theories underlying an arbitrary coset of the form $G_{K} \otimes G_{L} / G_{K+L}$. For the case of $S U(2)$, these field theories are the (fractional) super

\footnotetext{
${ }^{4}$ We recently received the preprints $[72,73]$, where RSOS $S$-matrices for cosets of the form $G_{1} \otimes G_{L} / G_{1+L}$ were also considered, and appear to be in agreement with the $S$-matrices in this section
} 
sine-Gordon theories and their restrictions [15]. The $S$-matrices (5.19), (5.34) and the Yangian (rational) limit of (5.19) are the basic building blocks for the $S$-matrices for the full series of cosets, the fractional super Toda theories, and for the currentcurrent perturbation of the WZW models at any level. The $S$-matrices for the cosets are a tensor product of two RSOS factors; they are obtained from a restriction of the fractional super Toda theories whose $S$-matrices are the tensor product of an RSOS factor and a trigonometric factor (5.19). The $S$-matrix for the massive WZW model is the tensor product of an RSOS factor and a Yangian invariant factor, where the RSOS factor is not present at level one. The arguments leading to these conclusions are based on the residual symmetries of the restricted models and their appropriate limits. The reader is referred to [11] for the details of the arguments.

Since the non-local currents completely characterize the $S$-matrices, we expect that they may also be used to constrain correlation functions. We believe that introducing the center into the symmetry algebra as was done in Sect. 4 is a promising new approach to this problem.

\section{Appendix A. Quantum Affine Algebras}

We review a few basic facts about quantum affine algebras.

Let $E_{1}, F_{1}, H_{1}$ denote a Chevalley basis for $s l(2)$. The algebra $\widehat{s l(2)}$ has an additional simple root with generators $E_{0}, F_{0}, H_{0}$. The affine Kac-Moody algebra $\widehat{s l}(2)$ is defined by the following relations:

$$
\begin{aligned}
{\left[H_{i}, E_{j}\right] } & =a_{i j} E_{j}, \\
{\left[H_{i}, F_{j}\right] } & =-a_{i j} F_{j}, \\
{\left[E_{i}, F_{j}\right] } & =\delta_{i j} H_{j}, \\
\left(\operatorname{Ad} E_{i}\right)^{1-a_{i j}}\left(E_{j}\right) & =0,
\end{aligned}
$$

where Ad is the adjoint action and $a_{i j}=\left(\begin{array}{cc}2 & -2 \\ -2 & 2\end{array}\right)$. The last relation in (A.1) is referred to as the Serre relation. The algebra has a central element $K=H_{0}+H_{1}$ called the level. When the center $K$ is zero the algebra is called the loop algebra and is isomorphic to $\operatorname{sl}(2) \otimes C\left(x, x^{-1}\right)$, where $C\left(x, x^{-1}\right)$ is an arbitrary function of a formal parameter $x$, referred to as the spectral parameter. Choosing $x^{n}, n \in Z$ as a basis for $C\left(x, x^{-1}\right)$, a zero-center representation of $\operatorname{sl}(2)$ is provided by $J_{n}^{a}=J^{a} x^{n}$ with $J^{a}$ forming a representation of $\widehat{s l(2)}$. In this representation, the Chevalley generators are,

$$
\begin{array}{ll}
E_{1}=x E_{+} ; & F_{1}=x^{-1} E_{-} ; \\
E_{0}=x E_{-} ; & F_{0}=x^{-1} E_{+} ; \\
H_{1}=H ; & H_{0}=-H,
\end{array}
$$

with $E_{ \pm}$and $H$ a Chevalley basis for the simple Lie algebra $s l(2)$,

$$
\begin{aligned}
{\left[H, E_{ \pm}\right] } & = \pm 2 E_{ \pm}, \\
{\left[E_{+}, E_{-}\right] } & =H .
\end{aligned}
$$


The representation (A.2) is in the so-called principal gradation. The gradation is only meaningful up to inner automorphisms of the Lie algebra $\operatorname{sl}(2)$. Another useful gradation is the homogeneous gradation with,

$$
\begin{array}{ll}
E_{1}=E_{+} ; & F_{1}=E_{-} ; \\
E_{0}=x^{2} E_{-} ; & F_{0}=x^{-2} E_{+} ; \\
H_{1}=H ; & H_{0}=-H .
\end{array}
$$

The relation between the two gradings for an element $a \in \widehat{\operatorname{sl}(2)}$ is,

$$
a_{\text {princ. }}(x)=\sigma a_{\text {homo. }}\left(x^{2}\right) \sigma^{-1},
$$

where $\sigma=x^{H / 2}$.

The algebra $s l_{q}(2)$ has the defining relations,

$$
\begin{aligned}
{\left[H_{i}, E_{j}\right] } & =a_{i j} E_{j}, \\
{\left[H_{i}, F_{j}\right] } & =-a_{i j} F_{j}, \\
{\left[E_{i}, F_{j}\right] } & =\delta_{i j} \frac{q^{H_{i}}-q^{-H_{i}}}{q-q^{-1}}, \\
\left(\operatorname{Ad}_{q} E_{i}\right)^{1-a_{i j}}\left(E_{j}\right) & =0,
\end{aligned}
$$

where $\mathrm{Ad}_{q}$ is a $q$-deformation of the adjoint action, first defined in [74]. The algebra $s l_{q}(2)$ is a Hopf algebra with comultiplication $\Delta$,

$$
\begin{aligned}
& \Delta\left(H_{i}\right)=H_{i} \otimes 1+1 \otimes H_{i}, \\
& \Delta\left(E_{i}\right)=E_{i} \otimes q^{-H_{i} / 2}+q^{H_{i} / 2} \otimes E_{i}, \\
& \Delta\left(F_{i}\right)=F_{i} \otimes q^{-H_{i} / 2}+q^{H_{i} / 2} \otimes F_{i} .
\end{aligned}
$$

The $\widehat{s l_{q}(2)}$ loop algebra in the principal or homogeneous gradation can be represented as in (A.2) or (A.4) where now $E_{ \pm}$and $H$ generate the $s_{q}(2)$ algebra:

$$
\begin{gathered}
{\left[H, E_{ \pm}\right]= \pm 2 E_{ \pm},} \\
{\left[E_{+}, E_{-}\right]=\frac{q^{H}-q^{-H}}{q-q^{-1}} .}
\end{gathered}
$$

The above structure is straightforwardly extended to the untwisted quantum affine algebras $\hat{G}_{q}$ with $G$ any simple Lie algebra. In this case the extended roots in the principle gradation are $E_{0}=x E_{-\vec{\theta}}, F_{0}=x^{-1} E_{\theta}$, where $\vec{\theta}$ is the highest root.

\section{Appendix B. Details on the Sine-Gordon $S$-Matrix}

Jimbo has shown that the solution $\breve{S}(x ; q)$ of Eqs. (3.39) can be written as follows,

$$
\breve{S}(x ; q)=\sigma_{21} \tilde{S}(x ; q) \sigma_{12}^{-1},
$$


where $x=x_{1} / x_{2}, \sigma_{12}=x_{1}^{H / 2} \otimes x_{2}^{H / 2}, \sigma_{21}=x_{2}^{H / 2} \otimes x_{1}^{H / 2}$ and,

$$
\begin{aligned}
\tilde{S}(x ; q) & =v(x)\left[x \breve{R}-x^{-1} \breve{R}^{-1}\right] \\
\breve{R} & =\left(\begin{array}{ccccc}
q & 0 & 0 & 0 \\
0 & \left(q-q^{-1}\right) & 1 & 0 \\
0 & 1 & 0 & 0 \\
0 & 0 & 0 & q
\end{array}\right)
\end{aligned}
$$

with $v(x)$ an arbitrary function of $x$. The matrix $\breve{R}$ plays a central role in the theory of the finite dimensional quantum group $s l_{q}(2)$. It satisfies the Hecke algebra,

$$
\begin{aligned}
(\breve{R}-q)\left(\breve{R}+q^{-1}\right) & =0, \\
\breve{R}_{21} \breve{R}_{32} \breve{R}_{21} & =\breve{R}_{32} \breve{R}_{21} \breve{R}_{32} .
\end{aligned}
$$

The Yang-Baxter equation for the $S$-matrix is a consequence of the Hecke algebra for $\breve{R}$.

The function $v(x)$ in (B.2) is fixed by the unitarity condition of the $S$-matrix:

$$
\tilde{S}(x ; q) \tilde{S}\left(x^{-1} ; q\right)=1 .
$$

Using the Hecke algebra relations (B.3), we have,

$$
\tilde{S}(x ; q) \tilde{S}\left(x^{-1} ; q\right)=v(x) v\left(x^{-1}\right) q^{-2}\left(1-x^{2} q^{2}\right)\left(1-x^{-2} q^{2}\right) .
$$

Therefore $v(x)$ is characterized by the equation,

$$
v(x) v\left(x^{-1}\right)=\frac{q^{2}}{\left(1-x^{2} q^{2}\right)\left(1-x^{-2} q^{2}\right)} .
$$

Crossing symmetry further requires $v(\theta)=v(i \pi-\theta)$, or

$$
v(x)=v\left(-\frac{1}{x q}\right)
$$

There exists a minimal solution to Eqs. (B.6) and (B.7) for $v(x)$. It is obtained iteratively as follows. One first tries $v(x)=q /\left(1-x^{2} q^{2}\right)$. This solves (B.6) but spoils the crossing symmetry. Crossing symmetry is restored by multiplying by $1 /\left(1-x^{-2}\right)$. Now unitarity is spoiled so we start again, the process never terminating. The result is,

$$
v(x)=\frac{q}{\left(1-x^{2} q^{2}\right)} \prod_{k=1}^{\infty}\left(\frac{1-x^{2} q^{4-4 k}}{1-x^{-2} q^{4-4 k}}\right)\left(\frac{1-x^{-2} q^{2-4 k}}{1-x^{2} q^{2-4 k}}\right) .
$$

Putting all these pieces together one verifies that (B.1) is the known $S$-matrix for sine-Gordon solitons.

\section{Appendix C. The Yangian Limit}

In this appendix we describe the connection between the $q \rightarrow-1$ limit of the quantum loop algebra and the Yangian symmetry. 
We first show how Yangian symmetry is generic to current-current perturbations of the $G$-invariant WZW models. Consider the action (5.27) in the instance that the WZW model is at level one. The currents satisfy the OPE

$$
J^{a}(z) J^{b}(w)=\frac{\delta^{a b}}{(z-w)^{2}}+f_{c}^{a b} \frac{J^{c}(w)}{z-w}+\cdots,
$$

and similarly for $\bar{J}$. Using this OPE in the equations of motion (2.10), we obtain

$$
\begin{aligned}
& \partial_{\bar{z}} J^{a}=\lambda\left(\partial_{z} \bar{J}^{a}+f_{b c}^{a} \bar{J}^{b} J^{c}\right), \\
& \partial_{z} \bar{J}^{a}=\lambda\left(\partial_{\bar{z}} J^{a}+f_{b c}^{a} J^{b} \bar{J}^{c}\right) .
\end{aligned}
$$

Define the gauge fields

$$
\begin{aligned}
& A_{z}=\frac{-2 \lambda}{1+\lambda} J, \\
& A_{\bar{z}}=\frac{-2 \lambda}{1+\lambda} \bar{J},
\end{aligned}
$$

where $J=\sum_{a} t^{a} J^{a}, \bar{J}=\sum_{a} t^{a} \bar{J}^{a}$, with $t^{a}$ a representation of the Lie algebra of $G$. The equations of motion (C.2) can be written as

$$
\begin{aligned}
\partial_{\bar{z}} A_{z}+\partial_{z} A_{\bar{z}} & =0, \\
{\left[\partial_{z}+A_{z}, \partial_{\bar{z}}+A_{\bar{z}}\right] } & =0 .
\end{aligned}
$$

The relations (C.4) are the foundation for the construction of some non-local charges that generate the Yangian symmetry $[5,6]$.

The on-shell realization of Yangian symmetry can be exhibited explicitly by taking the $\hat{\beta} \rightarrow \sqrt{2}$ limit of the algebraic equations characterizing the $S$-matrices (3.39), (5.12). We describe this computation for the case of $s l(2)$. There are two equivalent ways to write the $S$-matrix for the sine-Gordon model. The first one has been described above (3.39), (B.1); it corresponds to the quantum group parameter $q=-\exp (-i \pi / \gamma)$. In the second description, the $S$-matrix is still given by (B.1), but with the opposite sign quantum group parameter $q=\exp (-i \pi / \gamma)$. Both these $S$-matrices are crossing symmetric:

$$
\breve{S}_{a b}^{a^{\prime} b^{\prime}}(i \pi-\theta)=\kappa C_{a \bar{a}} \breve{S}_{\bar{a}^{\prime} b}^{\bar{a} b^{\prime}}(\theta) C^{\bar{a}^{\prime} a^{\prime}},
$$

where $C$ is the charge conjugation matrix and $C^{T}=\kappa C$. However they differ in the definition of charge conjugation; in the first case $C_{+_{-}}=C_{-+}=1$, whereas in the second case $C_{+-}=-C_{-+}=1$. Off shell, the choice of description amounts to a prescription for statistical Klein factors and is not dynamical; thus the two $S$-matrices describe the same theory. To take the limit $\hat{\beta} \rightarrow \sqrt{2}$, we choose the second description. In this limit, $x \approx 1+\varepsilon \theta$ and $q \approx 1-i \pi \varepsilon$, with $\varepsilon \rightarrow 0$.

The $S$-matrix in this limit becomes $\breve{S}\left(x_{1} / x_{2}\right)=f(\varepsilon) \breve{S}_{Y}\left(\theta_{1}-\theta_{2}\right)+\cdots$, with $f(\varepsilon)$ 
an irrelevant scalar function. The quantum group generators behave as follows:

$$
\begin{aligned}
& E^{ \pm}=J_{0}^{ \pm}+\varepsilon \frac{i \pi}{2} J_{1}^{ \pm}+\cdots, \\
& H_{0}=H, \\
& H_{1} \equiv \frac{i \pi}{2}\left[J_{0}^{+}, J_{1}^{-}\right] .
\end{aligned}
$$

We have defined the generator $H_{1}$ for later convenience.

The generators $J_{0}^{a}=J_{0}^{ \pm}, H_{0}$ satisfy the $s l(2)$ algebra:

$$
\left[H_{0}, J_{0}^{ \pm}\right]= \pm 2 J_{0}^{ \pm} ; \quad\left[J_{0}^{+}, J_{0}^{-}\right]=H_{0} .
$$

The generators $J_{1}^{a}=J_{1}^{ \pm}, H_{1}$ are $s l(2)$ intertwiners in the adjoint representation:

$$
\left[J_{0}^{a}, J_{1}^{b}\right]=f^{a b c} J_{1}^{c} \text {. }
$$

Jimbo has shown that a solution to $(3.39 \mathrm{~b})$ automatically satisfies $(3.39 \mathrm{a}, \mathrm{c})$. Equations $(3.39 \mathrm{a}, \mathrm{b})$ to zero ${ }^{\text {th }}$ order in $\varepsilon$ just imply the $s l(2)$ invariance of the limiting $\breve{S}_{Y}$ matrix:

$$
\breve{S}_{Y}\left(\theta_{12}\right)\left(J_{0}^{a} \otimes 1+1 \otimes J_{0}^{a}\right)=\left(J_{0}^{a} \otimes 1+1 \otimes J_{0}^{a}\right) \breve{S}_{Y}\left(\theta_{12}\right) .
$$

To first order in $\varepsilon(3.39 \mathrm{~b})$ reads

$$
\breve{S}_{Y}\left(\theta_{12}\right) \Delta_{\theta_{1} \theta_{2}}\left(J_{1}^{ \pm}\right)=\Delta_{\theta_{2} \theta_{1}}\left(J_{1}^{ \pm}\right) \breve{S}_{Y}\left(\theta_{12}\right)
$$

with $\theta_{12}=\theta_{1}-\theta_{2}$ and

$$
\Delta_{\theta_{1} \theta_{2}}\left(J_{1}^{ \pm}\right)=\left(J_{1}^{ \pm}+\frac{2 \theta_{1}}{i \pi} J_{0}^{ \pm}\right) \otimes 1+1 \otimes\left(J_{1}^{ \pm}+\frac{2 \theta}{i \pi} J_{0}^{ \pm}\right) \pm\left(J_{0}^{ \pm} \otimes H-H \otimes J_{0}^{ \pm}\right) .
$$

Equation (C.11) defines the comultiplication of $J_{1}^{ \pm}$. From its definition, one derives the comultiplication of $H_{1}$. The result is:

$$
\Delta_{\theta_{1} \theta_{2}}\left(H_{1}\right)=\left(H_{1}+\frac{2 \theta_{1}}{i \pi} H_{0}\right) \otimes 1+1 \otimes\left(H_{1}+\frac{2 \theta_{2}}{i \pi} H_{0}\right)-2\left(J_{0}^{+} \otimes J_{0}^{-}-J_{0}^{-} \otimes J_{0}^{+}\right) .
$$

The $\breve{S}_{Y}$ matrix is also $H_{1}$ invariant.

The comultiplication of the generators $J_{0}^{a}$ and $J_{1}^{a}$ can be written in the following synthesized form:

$$
\begin{aligned}
& \Delta_{\theta_{1} \theta_{2}}\left(J_{0}^{a}\right)=J_{0}^{a} \otimes 1+1 \otimes J_{0}^{a}, \\
& \Delta_{\theta_{1} \theta_{2}}\left(J_{1}^{a}\right)=\left(J_{1}^{a}+\frac{2 \theta_{1}}{i \pi} J_{0}^{a}\right) \otimes 1+1 \otimes\left(J_{1}^{a}+\frac{2 \theta_{2}}{i \pi} J_{0}^{a}\right)-\frac{1}{2} f^{a b c} J_{0}^{b} \otimes J_{0}^{c} .
\end{aligned}
$$

The exchange relations (C.9) and (C.10), and the comultiplication (C.13), are those of the $\operatorname{sl}(2)$ Yangian [2].

The solution to (C.9), (C.10), for the fundamental representation, is $\breve{S}_{Y}(\theta) \propto i \pi-\theta P$, where $P$ is the permutation operator: $P \cdot(u \otimes v)=v \otimes u$. 


\section{Appendix D. Generalized Parafermions and Their Braidings}

Here we make contact between the braiding relations (3.16) and those of disorder fields and generalized parafermions $[16,17]$.

Disorder fields and generalized parafermions are in general non-local fields which reflect the group invariance of the model. We denote by $\mathscr{G}$ the group. In a lattice formulation the disorder fields are defined by introducing a disorder line along which the spins of the lattice model are flipped by acting with an element $g \in \mathscr{G}$. The group invariance of the hamiltonian ensures that the exact shape of the disorder line is irrelevant up to possible topological obstructions. Thus disorder fields, which we denote by $\mu_{g}(x, t), g \in \mathscr{G}$, are labeled by group elements. On the contrary, the spin fields, which we denote by $\sigma^{\rho}(x, t)$, are labeled by the representations $\rho$ of the group, $\rho \in \operatorname{Rep}(\mathscr{G})$. The spin fields are local fields, which in the lattice description correspond to the insertions of the matrix representing the group. The generalized parafermions are the product of a disorder field with a spin field (the product is to be regularized in the continuum). We denote them by $\Psi_{g}^{\rho}(x, t)=$ $\mu_{g}(x, t) \sigma^{\rho}(x, t)$, with $g \in \mathscr{G}$ and $\rho \in \operatorname{Rep}(\mathscr{G})$; it takes values in the representation $\rho$. The braiding relations for the generalized parafermions are well-known,

$$
\begin{array}{r}
\left(\Psi_{g_{1}}^{\rho_{1}}(x, t) \otimes 1\right)\left(1 \otimes \Psi_{g_{2}}^{\rho_{2}}(y, t)\right)=\left(1 \otimes \rho_{2}\left(g_{1}\right) \underset{g_{1} g_{2} g_{1}^{-1}}{\rho_{2}}(y, t)\right)\left(\underset{g_{1}}{\rho_{1}}(x, t) \otimes 1\right) \\
\text { for } \quad x>y .
\end{array}
$$

For $g=1$ or $\rho=1,(\mathrm{D} .1)$ gives the braiding relations for the disorder fields and for the spin fields. Note that in general there is no natural pairing between the group $\mathscr{G}$ and $\operatorname{Rep}(\mathscr{G})$.

Let us now specialize to the abelian group $\alpha Z$ where $\alpha$ is any fixed real number. Elements of $\alpha Z$ are $\{\alpha n\}$, where $n$ is any integer. (For the application to Sect. 3d, $\alpha=\frac{1}{\hat{\beta}}$.) The unitary representation of $\alpha Z$ are one-dimensional and are labeled by a real number $r$. We denote them by $\rho_{r}, r \in R$, with $\rho_{r}(a)=e^{i \pi r a}$, for $a \in \alpha Z$. Hence for the group $\alpha Z$ there are natural embeddings of $\mathscr{G}$ into $\operatorname{Rep}(\mathscr{G})$ given by $a \in \alpha Z \rightarrow$ $[a] \equiv \rho_{a} \in \operatorname{Rep}(\alpha Z)$. This allows us to introduce a particular set of parafermions defined by ${ }^{*}$

$$
\begin{aligned}
& \Psi_{a}(x, t)=\mu_{a}(x, t) \sigma^{[a]}(x, t), \\
& \bar{\Psi}_{a}(x, t)=\mu_{-a}(x, t) \sigma^{[a]}(x, t) .
\end{aligned}
$$

These two parafermions only differ by the change $a \rightarrow-a$ in the disorder fields. The braiding relations (D.1) then reduce to,

$$
\begin{aligned}
& \Psi_{a}(x, t) \Psi_{b}(y, t)=e^{ \pm i \pi a b} \Psi_{b}(y, t) \Psi_{a}(x, t) ; \text { for } \quad x \gtrless y ; \\
& \bar{\Psi}_{a}(x, t) \bar{\Psi}_{b}(y, t)=e^{\mp i \pi a b} \bar{\Psi}_{b}(y, t) \bar{\Psi}_{a}(x, t) ; \text { for } \quad x \gtrless y ; \\
& \Psi_{a}(x, t) \bar{\Psi}_{b}(y, t)=e^{i \pi a b} \bar{\Psi}_{b}(y, t) \Psi_{a}(x, t) ; \quad \forall x, y .
\end{aligned}
$$

These relations are the same as in (3.16). Therefore, we have,

$$
\Psi_{a}(x, t)=\exp (i a \phi(x, t)) ; \quad \bar{\Psi}_{a}(x, t)=\exp (i a \bar{\phi}(x, t)) .
$$

All fields, including the disorder fields and the spin fields, are generated from these. 
Acknowledgements. We would like to thank A. Belavin, G. Felder, M. Olshanetsky, F. Smirnov, and A. Zamolodchikov for discussions. A.L. would like to thank the Saclay group and the Aspen Center of Physics for hospitality in the course of this work. This work is supported in part by the National Science Foundation.

\section{References}

1. Coleman, S., Mandula, J.: Phys. Rev. 159, 1251 (1967)

2. Drinfel'd, V. G.: Sov. Math. Dokl. 32, 254 (1985); Sov. Math. Dokl. 36, 212 (1988)

3. Jimbo, M.: Math. Phys. 10, 63 (1985); Lett. Math. Phys. 11, 247 (1986); Commun. Math. Phys. 102, 537 (1986)

4. Faddeev, L. D., Reshetikhin, N. Yu., Takhtajan, L. A.: Quantization of Lie Groups and Lie Algebras 1989 preprint

5. Lüscher, M.: Nucl. Phys. B135, 1 (1978)

6. Bernard, D.: Commun. Math. Phys. 137, 191 (1991)

7. de Vega, H. J., Eichenherr, H., Maillet, J. M.: Nucl. Phys. B240, 377 (1984); Commun. Math. Phys. 92, 507 (1984)

8. Zamolodchikov, A. B., Zamolodchikov, Al. B.: Ann. Phys. 120, 253 (1979)

9. Coleman, S.: Phys. Rev. D11, 2088 (1975)

10. Mandelstam, S.: Phys. Rev. D11, 3026 (1976)

11. Ahn, C., Bernard, D., LeClair, A.: Nucl. Phys. B346, 409 (1990)

12. Hollowood, T.: A Quantum Group Approach to Constructing Factorizable S-Matrices: Oxford Preprint OUTP-90-15P, June 1990

13. Sklyanin, E.'K., Takhtadzhyan, L. A., Faddeev, L. D.: Theor. Math. 40, 688 (1980)

14. Faddeev, L.: Les Houches Lectures 1982, Elsevier Science Publishers (1984)

15. Bernard, D., LeClair, A.: The Fractional Super Sine-Gordon Models. Phys. Lett. B247, 309 (1990)

16. Kadanoff, L. P., Ceva, H.: Phys. Rev. B3, 3918 (1971); Fradkin, E., Kadanoff, L. P.: Nucl. Phys. B170, 1 (1980)

17. Zamolodchikov, A. B., Fateev, V. A.: Sov. Phys. JETP 63, 913 (1986)

18. Fröhlich, J.: Statistics of Fields, the Yang-Baxter Equation, and the Theory of Knots and Links. Cargese Lectures 1987; 't Hooft G. et al. (eds.). New York: Plenum Press

19. Fredenhagen, K., Rehren, K. H., Schroer, B.: Commun. Math. Phys. 125, 201 (1989)

20. Woronowicz, S. L.: Commun. Math. Phys. 122, 125 (1989)

21. Bernard, D.: Quantum Lie Algebras and Differential Calculus on Quantum Groups. Saclay preprint SPhT-90-124; A Propos du Calcul Differentiel sur les Groupes Quantiques (in French), Saclay preprint SPhT-90-119

22. Wess, J., Zumino, B.: Covariant Differential Calculus on the Quantum Hyperplane. preprint CERN-TH-5697/90, April 1990

23. Bernard, D., Felder, G., LeClair, A.: Non-local Conserved Charges: The Lattice Approach

24. Zamolodchikov, A. B.: Int. J. Mod. Phys. A4, 4235 (1989); Adv. Studies Pure Math. 19, 641 (1989)

25. Witten, E., Olive, D.: Phys. Lett. B78, 97 (1978)

26. Ginsparg, P.: Nucl. Phys. B295, 153 (1988)

27. Smirnov, F. A.: Commun. Math. Phys. 132, 415 (1990)

28. Witten, E.: Commun. Math. Phys. 92, 455 (1984); Polyakov, A., Wiegmann, P. B.: Phys. Lett. B141, 223 (1984); Faddeev, L. D., Reshetikhin, N. Yu.: Ann. Phys. 167, 227 (1986)

29. Goddard, P., Nahm, W., Olive, D., Schwimmer, A.: Commun. Math. Phys. 107, 179 (1986); Bernard, D., Thierry-Mieg, J.: Commun. Math. Phys. 111, 181 (1987); Goddard, P., Olive, D.: Int. J. Mod. Phys. A1, 303 (1986); Frenkel, I. B., Kac, V. G.: Inv. Math. 62, 23 (1980)

30. Halpern, M. B.: Phys. Rev. D12, 1684 (1976)

31. Knizhnik, V. G., Zamolodchikov, A. B.: Nucl. Phys. B297, 83 (1984)

32. Banks, T., Horn, D., Neuberger, H.: Nucl. Phys. B108, 119 (1976)

33. LeClair, A.: Phys. Lett. 230B, 103 (1989); Cornell preprint CLNS 89/955, Oct. 1989. Integrable Restrictions of Quantum Soliton Theory and Minimal Conformal Series. To be published 
in the proceedings of the 1989 Banff workshop on Physics, Geometry, and Topology. Lee, H. C. (ed.). New York: Plenum Press

34. Smirnov, F. A.: Int. J. Mod. Phys. A4, 4213 (1989)

35. Bernard, D., LeClair, A.: Nucl. Phys. B340, 721 (1990)

36. Reshetikhin, N. Yu., Smirnov, F.: Commun. Math. Phys. 131, 157 (1990)

37. Eguchi, T., Yang, S.-T.: Phys. Lett. B235, 282 (1990)

38. Belavin, A. A., Polyakov, A. M., Zamolodchikov, A. B.: Nucl. Phys. B241, 333 (1984)

39. Friedan, D., Qiu, Z., Shenker, S.: Phys. Rev. Lett. 52, 1575 (1984)

40. Dotsenko, V., Fateev, V.: Nucl. Phys. B240, 312 (1984)

41. Felder, G.: Nucl. Phys. B317, 215 (1989)

42. Pasquier, V.: Commun. Math. Phys. 118, 355 (1988)

43. Kirillov, A. N., Reshetikhin, N.: LOMI preprint E-9-88, published in the Proceedings of Marseille Conference. Kac, V. (ed.). Singapore: World Scientific

44. Keller, G.: Fusion Rules of $U_{q}(s l(2, C)), q^{m}=1$. Max-Planck-Institut preprint MPI-PAE/Pth 47/90, July 1990

45. Goddard, P., Kent, A., Olive, D.: Phys. Lett. 152B, 88 (1985)

46. Tsuchiya, A., Kanie, Y.: Lett. Math. B, 303 (1987)

47. Moore, G., Seiberg, N.: Phys. Lett. B212, 451 (1988); Commun. Math. Phys. 123, 177 (1989)

48. Alvarez-Gaumé, L., Gomez, C., Sierra, G.: Phys. Lett. B220, 142 (1989); Nucl. Phys. B330, 347 (1990)

49. Felder, G., Fröhlich, J., Keller, G.: Commun. Math. Phys. 124, 647 (1989)

50. Babelon, O.: Phys. Lett. B215, 523 (1988)

51. Gervais, J.-L.: Commun. Math. Phys. 130, 257 (1990)

52. Smirnov, F., Takhtajan, L.: Towards a Quantum Liouville Theory with $c>1$. Colorado preprint 1990

53. Gomez, C., Sierra, G.: Phys. Lett. B240, 149 (1990); Nucl. Phys. B352, 791 (1991)

54. Korepin, y. E.: Teor. Mat. Fiz. 41, 169 (1979)

55. Babelon, O., Bonora, L.: Phys. Lett. B244, 220 (1990)

56. Grisaru, M. T., Lerda, A., Penati, S., Zanon, D.: Nucl. Phys. B346, 264 (1990)

57. Jimbo, M.: Int. Mod. Phys. A4, 3759 (1989)

58. Karowski, M., Thun, H. J.: Nucl. Phys. B190, 61 (1981)

59. Ogievetsky, E., Reshetikhin, N. Yu., Wiegmann, P.: Nucl. Phys. B280 [FS18], 45 (1987)

60. Kulish, P. P., Reshetikhin, N. Yu., Sklyanin, E. K.: Lett. Math. Phys. 5, 393 (1981)

61. Ogievetsky, E., Wiegmann, P.: Phys. Lett. 168B, 360 (1986)

62. Zamolodchikov, A. B.: Sov. J. Nucl. Phys. 46, 1090 (1987)

63. Bilal, A., Gervais, J.-L.: Phys. Lett. B206, 412 (1988)

64. Fateev, V., Lykanov, S.: Int. J. Mod. Phys. A3, 507 (1988)

65. Eguchi, T., Yang, S.-K.: Phys. Lett. B224, 373 (1989)

66. Hollowood, T., Mansfield, P.: Phys. Lett. B226, 73 (1989)

67. Smirnov, F. A.: Int. J. Mod. Phys. A6, 1253 (1991)

68. Date, E., Jimbo, M., Kuniba, A., Miwa, T., Okado, M.: Nucl. Phys. B290, 231 (1987)

69. Jimbo, M., Kuniba, A., Miwa, T., Okado, M.: Commun. Math. Phys. 119, 543 (1988)

70. Fateev, V. A., Zamolodchikov, A. B.: Int. J. Mod. Phys. A5, 1025 (1990); Christe, P., Mussardo, G.: Nucl. Phys. B330, 465 (1990); Sotkov, G., Zhu, C.-J.: Phys. Lett. B229, 391 (1989); Klassen, T. R., Melzer, E.: Nucl. Phys. B338, 485 (1990)

71. Braden, H. W., Corrigan, E., Dorey, P. E., Sasaki, R.: Nucl. Phys. B338, 698 (1990)

72. de Vega, H. J., Fateev, V. A.: Int. J. Mod. Phys. A6, 3221 (1991)

73. Nakatsu, T.: Nucl. Phys. B356, 499 (1991)

74. Bernard, D.: Lett. Math. Phys. 17, 239 (1989) 